\title{
From Spinon Band Topology to the Symmetry Quantum Numbers of Monopoles in Dirac Spin Liquids
}

\author{
Xue-Yang Song, ${ }^{1}$ Yin-Chen He, ${ }^{2,1}$ Ashvin Vishwanath, ${ }^{1}$ and Chong Wang ${ }^{2,1}$ \\ ${ }^{1}$ Department of Physics, Harvard University, Cambridge, Massachusetts 02138, USA \\ ${ }^{2}$ Perimeter Institute for Theoretical Physics, Waterloo, Ontario N2L 2Y5, Canada
}

(Received 9 April 2019; revised manuscript received 22 October 2019; accepted 20 November 2019; published 12 February 2020)

\begin{abstract}
The interplay of symmetry and topology has been at the forefront of recent progress in quantum matter. Here, we uncover an unexpected connection between band topology and the description of competing orders in a quantum magnet. Specifically, we show that aspects of band topology protected by crystalline symmetries determine key properties of the Dirac spin liquid (DSL), which can be defined on the honeycomb, square, triangular, and kagome lattices. At low energies, the DSL on all of these lattices is described by an emergent quantum electrodynamics $\left(\mathrm{QED}_{3}\right)$ with $N_{f}=4$ flavors of Dirac fermions coupled to a $U(1)$ gauge field. However, the symmetry properties of the magnetic monopoles, an important class of critical degrees of freedom, behave very differently on different lattices. In particular, we show that the lattice momentum and angular momentum of monopoles can be determined from the charge (or Wannier) centers of the corresponding spinon insulator. We also show that for DSLs on bipartite lattices, there always exists a monopole that transforms trivially under all microscopic symmetries owing to the existence of a parent $S U(2)$ gauge theory. We connect our results to generalized Lieb-Schultz-Mattis theorems and also derive the time-reversal and reflection properties of monopoles. Our results indicate that recent insights into free-fermion band topology can also guide the description of strongly correlated quantum matter.
\end{abstract}

DOI: 10.1103/PhysRevX.10.011033

\section{INTRODUCTION}

Quantum spin liquids represent a class of exotic quantum phases of matter beyond the traditional Landau symmetry-breaking paradigm. Besides being conceptually meaningful and experimentally relevant on their own [1-3], they are also connected to various deep problems ranging from high-temperature superconductivity to topological order and strongly coupled gauge theories, to name a few $[4,5]$.

A particularly important quantum spin liquid in two spatial dimensions is the Dirac spin liquid (DSL) [4,6-12]. The DSL is described by fermionic spinons-emergent particles carrying spin-1/2 - whose dispersion at low energies is described by the massless Dirac equation. These Dirac spinons interact with an emergent photon $[U(1)$ gauge field], an effective field theory known as $\mathrm{QED}_{3}$. At low energy and long wavelength, the effective theory is described by the continuum Lagrangian

Published by the American Physical Society under the terms of the Creative Commons Attribution 4.0 International license. Further distribution of this work must maintain attribution to the author(s) and the published article's title, journal citation, and DOI.
Subject Areas: Condensed Matter Physics

$$
\mathcal{L}=\sum_{i=1}^{4} \bar{\psi}_{i}(i \not \supset-\not \supset) \psi_{i}+\frac{1}{4 e^{2}} f_{\mu \nu}^{2},
$$

where the first term describes Dirac fermions $\psi$ minimally coupled with an emergent $U(1)$ gauge field $a$, and the second term is a standard Maxwell term for the gauge field $a$. This spin-liquid state was originally discussed on the square lattice in the context of high- $T_{c}$ cuprates (as a candidate state of the "pseudogap" regime) [4] and as a "mother state" of different competing orders [13]. On the kagome lattice, the DSL is a candidate ground state for the Heisenberg antiferromagnet $[8,9,14]$, as supported by recent density matrix renormalization group calculations $[10,12]$, and may potentially be relevant for experimental systems such as herbertsmithite [15,16], although gapped spin liquids have also been proposed in this context. On the triangular lattice, a spin liquid is observed in density matrix renormalization group studies when a small second neighbor spin coupling $J_{2}$ is added in the range $0.07<J_{2} / J_{1}<$ 0.15 [17], and variational Monte Carlo simulation suggested it to be a Dirac spin liquid [11]. As predicted for a Dirac spin liquid, a chiral spin liquid is obtained in this parameter range as soon as a time-reversal symmetrybreaking perturbation is applied [18,19]. Further support comes from recent lattice gauge theory simulations, which 
reported that $\mathrm{QED}_{3}$, even with a relatively small number of fermion flavors, may exhibit a stable critical phase, at least when symmetry lowering perturbations and monopoles were absent (sometimes called "noncompact" $\mathrm{QED}_{3}$ ) $[20,21]$. This raises the remarkable possibility that the DSL may be realized as a stable phase (or perhaps a critical point; see Ref. [22]) on the triangular lattice. Intriguingly, quantum-spin-liquid materials candidates have recently emerged on the triangular lattice $[23,24]$. In other words, Dirac spin liquids appear to be relevant for a variety of candidate spin-liquid systems and therefore certainly deserve to be understood thoroughly.

To make progress, however, one really needs a rigorous understanding of monopoles of the $U(1)$ gauge field [25-27]. These are flux-insertion operators, which we define more precisely in Sec. II, and they form an important class of excitations (more accurately, critical fluctuations) in the DSL. Despite the long history of the theory of DSL (over two to three decades), a fundamental aspect of the monopoles, namely, their quantum numbers under the microscopic symmetries (lattice, time-reversal, etc.), is still an unresolved issue. This is an important issue for the following three reasons:

(1) Stability of DSL: If a monopole transforms trivially under all physical symmetries, it should be added as an allowed perturbation in the effective theory, Eq. (1). If this monopole is also relevant in the renormalization-group ( $R G)$ sense, it will lead to instabilities of the DSL $[25,27,28]$.

(2) Universal properties: In the absence of monopole perturbations that are symmetry allowed and RG relevant, the DSL can be realized as a stable gapless phase. The monopoles transform under the action of physical symmetries. The most relevant monopoles (in the RG sense) pick our specific representations of the symmetry group, which lead to a class of important critical fluctuations. For example, some monopoles may behave as critical spin fluctuations at a specific lattice momentum point, which may have observable consequences in, for example, spectroscopic measurements. Understanding the symmetry properties of the monopoles is therefore crucial in the DSL phase.

(3) Symmetry-breaking orders: The properties of the monopoles also decide the nature of other, more conventional, phases in proximity to the DSL [28]. Essentially, they serve as the natural "order parameters" of symmetry-breaking phases when the DSL is destabilized (for example, by strong perturbations).

In other words, we have to understand the properties of monopoles because monopoles represent the most important aspect of gauge fluctuations beyond the mean-field approximation. In the simpler case of a semiclassical theory of fluctuating Néel order, or equivalently a theory based on bosonic spinons (Schwinger bosons) coupled to a $U(1)$ gauge field that naturally appears on bipartite lattices, the lattice-symmetry properties of monopoles were calculated in Refs. [29,30]. This played an important role in predicting valence bond solid (VBS) order as a competing singlet state, and in the development of deconfined criticality [31,32], of the Néel-VBS phase transition. However, for fermionic spinons that provide an intrinsically quantum mechanical description, such an analytic understanding is still absent. Some progress was made in Ref. [33], in which the monopole quantum numbers on the square lattice were shown to be constrained by group-theoretic considerations [34] and were eventually calculated numerically. Subsequently, an analysis of the honeycomb [35] and kagome [9] lattices was also initiated. We report the numerical computation of monopole quantum numbers for several symmetries on these lattices in a parallel paper [28] and also discuss consequences for the stability and the phenomenology of the DSL. In contrast, in this work, we uncover a close and unexpected connection between the symmetry properties of monopoles and fermion band topology. This connection allows us to build on recent progress understanding band topology protected by crystalline symmetries, to develop a systematic analytical approach to calculate the monopole symmetry quantum numbers on essentially any lattice-although we focus on the physically relevant ones, including square, honeycomb, triangular, and kagome lattices. Armed with this deeper understanding and analytical machinery, we are able to obtain a complete understanding of the symmetry action on monopoles.

Our new understanding was enabled by developments in the theories of topological insulators and topological crystalline insulators over the past decade. Essentially, the symmetry properties of the monopoles are fixed by the "band topology" of the underlying fermionic spinons. By establishing the precise connection between band topology and monopole quantum numbers, the latter can be calculated using the technology of topological band theory. An analogous approach has long been used to determine monopole quantum numbers associated with continuous, on-site symmetries. For example, when fermions fill a Chern band, a Chern-Simons term is generated that represents charge-flux attachment. Similarly, the $S_{z}$ spin is carried by the monopole (a flux quantum) in the presence of a quantized spin Hall conductance [35]. In this work, we leverage the full power of topological band theory to determine monopole quantum numbers to include lattice symmetries and time reversal.

It turns out that the monopoles' lattice momenta and angular momenta (the most challenging part of the problem) is related to an old concept in band theory: the charge (or Wannier) centers of an occupied band. The basic idea is extremely simple: If a charge sits at a point in space (the Wannier center), a monopole (magnetic flux) picks up a Berry phase when moving around it. Recent developments [36-39], especially the clarification of the notion of "fragile 
topology," enabled us to calculate the charge centers even when there is an obstruction to obtaining localized Wannier states. In fact, this is a frequent occurrence in the states we discuss; nevertheless, we are able to obtain the location of charge centers efficiently, which then feature sites with both negative and positive charges. We note that a similar calculation has been applied to a particular spinon meanfield theory on the square lattice in Ref. [40], where the charges could be localized on lattice sites.

We also see that monopoles behave very differently on bipartite (honeycomb and square) and nonbipartite (triangular and kagome) lattices: On bipartite lattices, there always exists a monopole that transforms trivially under all the microscopic symmetries, making it an allowed perturbation to the theory, thereby likely destabilizing the DSL; on nonbipartite lattices, this does not happen, at least not in the examples we consider. The difference can be traced to the fact that on bipartite lattices, one can continuously tune the DSL state to another spin-liquid state with an $S U(2)$ [instead of a $U(1)$ ] gauge group. This connection leads to a different, and simpler, way of calculating monopole quantum numbers on bipartite lattices, with results that are consistent with the band topology approach.

\section{A. Summary of results}

Here, we summarize our main results and physical pictures without going into technical details. This section also serves as a map for the rest of this paper.

(1) The symmetry quantum numbers carried by monopoles are determined by the band topology (or symmetry-protected-topological order) of the underlying spinon mean-field theory. For time-reversal and reflection symmetries, the corresponding topological invariants are that of the "strong" topological insulators. For example, a Kane-Mele topological insulator makes the corresponding monopole creation operator odd under time-reversal transformation. This result is very similar to the "quantum spin Hall" response, where an $S_{z}$ spin is trapped in a monopole (flux quantum). This case is discussed in detail in Sec. III.

(2) For lattice translation and rotation symmetries, the corresponding topological invariants of the spinon bands are related to the old concept of charge (or Wannier) centers. The basic idea is illustrated with the example in Fig. 1: Consider an "atomic limit" of an insulator that can be represented as static (nonfluctuating) charges sitting at some real-space points (not necessarily the lattice sites), such as that illustrated in Fig. 1. A flux quantum (a.k.a. monopole) moving around any point in space sees the charge sitting at that point as a source of the Aharonov-Bohm phase, which leads to an angular momentum $l=e \times m$ with respect to that point, where $e$ is the charge at the rotation center and $m$ is the strength of the flux (normalized to 1 for a $2 \pi$

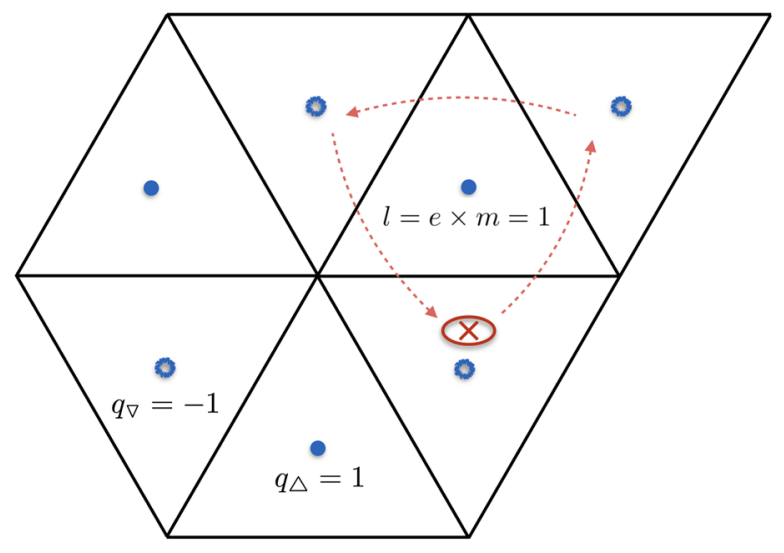

FIG. 1. An example showing how charge centers of a spinon band determine lattice-symmetry quantum numbers (like the angular momentum) of a monopole (a flux quantum).

flux)-semiclassically, this result is simply the angular momentum stored in the gauge field. For the example in Fig. 1, we have $l_{\Delta}=1$ and $l_{\nabla}=-1$ for the $C_{3}$ rotations around the centers of upper and lower triangles, respectively. The difference between angular momenta around different points determines the linear crystalline momentum since a lattice translation can be obtained through opposite rotations around different points. In the example of Fig. 1, this means that the horizontal momentum of the monopole $k_{x}=(2 \pi / 3)\left(l_{\nabla}-l_{\Delta}\right)=(2 \pi / 3)(\bmod$ $2 \pi)$. For a given spinon band structure, the charge centers can be calculated efficiently using modern topological band theory [36-39]. More details of this approach are discussed in Secs. V and VI.

(3) It turns out that on bipartite lattices (like the square and honeycomb), there is always a $2 \pi$ monopole that transforms trivially under all physical symmetries. This case is always true whenever the corresponding lattice gauge theory can be smoothly tuned to a particle-hole symmetric point, which happens for all the theories on bipartite lattices we consider in this work. The underlying reason is that at the particlehole symmetric point, the system is really described by an emergent $S U(2)$ gauge theory. In Sec. IV A, we discuss this point in detail and show that the existence of a symmetry-trivial monopole alone is essentially sufficient to determine the quantum numbers of other monopoles. This case offers a method to calculate monopole quantum numbers on bipartite lattices that is very different from the charge (Wannier) center approach, yet they produce exactly the same results on bipartite lattices. In Sec. IV B, we show that the particle-hole symmetry on bipartite lattices offers yet another way to calculate monopole quantum numbers by combining different reflection symmetries-this approach also yields results that agree with the other two approaches mentioned above. 
(4) In contrast to the bipartite lattices, on nonbipartite lattices (triangular and kagome), we find that all the $2 \pi$ monopoles are nontrivial under some physical symmetries. This case leads to a dichotomy between Dirac spin liquids on bipartite and nonbipartite lattices: On a bipartite lattice, the DSL is likely unstable due to the existence of a symmetry-allowed monopole perturbation, and the eventual fate of the DSL is likely a symmetry-breaking state such as the Néel or VBS state; on nonbipartite lattices, however, the DSL is potentially realizable as a stable gapless phase. This result is also consistent with recent numerical works, where all the DSL candidates were found on nonbipartite lattices. When a DSL is stabilized as a gapless phase on a nonbipartite lattice, the monopoles form an important class of gapless degrees of freedom. In the examples we consider, they behave as fluctuating coplanar magnetic orders and VBS orders. This case leads to observable consequences in spectroscopic measurements (e.g., neutron scattering). For example, on the triangular lattice, this leads to a fluctuating spin mode at the zone-corner momentum points $\mathbf{K}, \mathbf{K}^{\prime}$ - these momenta come precisely from monopoles influenced by the band topology in Fig. 1 and cannot be understood simply from the correlation functions of the spinons. Furthermore, when the DSL is destroyed through a quantum phase transition to a conventional symmetry-breaking phase, the monopoles become the natural order parameters and therefore decide the patterns of symmetry breaking. For example, on the triangular lattice, one of the phases in proximity to the DSL is the famous 120-degree coplanar magnetic order-precisely the $\mathbf{K}, \mathbf{K}^{\prime}$ magnetic fluctuations mentioned above. We discuss these points with some illustrative examples in Sec. VIII, specifically focusing on square and triangular lattices. In Ref. [28], we perform a more complete analysis on all four lattices considered in this work and discuss more physical consequences in detail.

(5) The possible low-energy theories of a spin system are heavily constrained by the Lieb-Schultz-Mattis (LSM) type of theorems [41-43]-for example, a trivially gapped ground state cannot emerge out of a system with spin-1/2 per lattice unit cell with translation symmetry. In the continuum field theory descriptions, these constraints are manifested as symmetry anomalies [44-47]. We show in Sec. IX that the monopole quantum numbers obtained in this work are indeed consistent, in a nontrivial way, with LSM-type anomalies. In particular, we show that LSM anomalies essentially fix the actions of inversion symmetry on the monopoles.

(6) The methods used in this work, especially the charge center approach, can certainly be generalized to other types of spin liquids. In Sec. X, we briefly outline how to apply our method to $U(1)$ spin liquids in three dimensions, which is of interest recently due to potential realizations in quantum spin ice materials [2].

Next, in Sec. II, we review some basic aspects of $U(1)$ Dirac spin liquids and define precisely the problem of monopole quantum numbers.

\section{GENERALITIES}

\section{A. $U(1)$ Dirac spin liquid and monopole operators}

We start with the standard parton decomposition of the spin- $1 / 2$ operators on the lattice,

$$
\mathbf{S}_{i}=\frac{1}{2} f_{i, \alpha}^{\dagger} \sigma_{\alpha \beta} f_{i, \beta},
$$

where $f_{i, \alpha}$ is a fermion (spinon) on site $i$ with spin $\alpha \in\{\uparrow, \downarrow\}$ and $\sigma$ are Pauli matrices. This rewriting is exact if we stay in the physical Hilbert space, defined by the constraint $\sum_{\alpha} f_{i, \alpha}^{\dagger} f_{i, \alpha}=1$. We now relax the constraint and allow the fermionic spinons to hop on the lattice (for more details, see Ref. [5]), according to a mean-field Hamiltonian

$$
H_{\mathrm{MF}}=-\sum_{i j} f_{i}^{\dagger} t_{i j} f_{j} .
$$

At the mean-field level, the spinons are just like the usual spinful fermions. There is a gauge redundancy $f_{i} \rightarrow e^{i \alpha_{i}} f_{i}$ in the parton decomposition, Eq. (2), which results in the emergence of a dynamical $U(1)$ gauge field $a_{\mu}$ that couples to the fermions $f$. Each site carries a gauge charge $q_{i}=\sum_{\alpha} f_{i, \alpha}^{\dagger} f_{i, \alpha}-1$. In the strong coupling limit where the gauge field simply enforces a constraint $q_{i}=0$ on each site, the physical spin Hilbert space is recovered. However, if the gauge coupling does not flow to infinity in the lowenergy limit (this can almost be viewed as the definition of a spin-liquid phase), the gauge charge only needs to vanish on average, $\langle q\rangle=0$.

We now arrange the hopping amplitudes $t_{i j}$ in a way so that the spinons form four Dirac cones at low energy: two Dirac valleys per spin, as required by fermion doubling. For example, on the honeycomb lattice, one can just take a uniform, real, and nonbipartite hopping, and two Dirac valleys will generically appear. The nonbipartite nature (second-neighbor hopping) is needed to make sure that the gauge group is $U(1)$ rather than $S U(2)$ [5] - this will play an important role later in Sec. IV A. The relevant mean-field Hamiltonians on square, honeycomb, triangle, and kagome lattices are described in detail in the Appendix A.

The dispersion of the mean-field Hamiltonian, Eq. (3), has four gapless Dirac cones at half-filling (e.g., see Fig. 9), two for each spin species. Taking the continuum limit, the $U(1)$ Dirac spin liquids in the low-energy, long-wavelength 
(IR) limit can effectively be described by the following (Euclidean) Lagrangian:

$$
\mathcal{L}=\sum_{i=1}^{4} \bar{\psi}_{i} i \not_{a} \psi_{i}+\frac{1}{4 e^{2}} f_{\mu \nu}^{2},
$$

where $\psi_{i}$ is a two-component Dirac fermion and $a$ is a dynamical $U(1)$ gauge field. We choose $\left(\gamma_{0}, \gamma_{1}, \gamma_{2}\right)=$ $\left(\mu^{2}, \mu^{3}, \mu^{1}\right)$, where $\mu$ are Pauli matrices. This theory is also known as $\mathrm{QED}_{3}$, with $N_{f}=4$. The theory flows to strong coupling at an energy scale below $e^{2}$, and its ultimate IR fate is not completely known. In this work, we assume that when monopole instantons are suppressed (to be explained in more detail below), this QED theory flows to a stable critical fixed point in the IR, as supported by recent numerics $[20,21]$.

Naively, there is a conserved current in the theory,

$$
j_{\mu}=\frac{1}{2 \pi} \epsilon_{\mu \nu \lambda} \partial_{\nu} a_{\lambda},
$$

that corresponds to a global $U(1)$ symmetry sometimes called $U(1)_{\text {top }}$. The conserved charge is simply the magnetic flux of the emergent $U(1)$ gauge field. One can then define operators that carry this global $U(1)_{\text {top }}$ charge, i.e., operators that create or annihilate the total gauge flux by $2 \pi$, and these operators are known as monopoles in the literature. We denote a monopole operator by $\mathcal{M}$. One can think of $\mathcal{M}$, in the path-integral formulation, as a point in space-time surrounded by a $2 \pi$ flux. In the Hamiltonian formulation, we can think of it as an operator,

$$
\mathcal{M}=\sum_{n}\left|\Omega_{2 \pi n}\right\rangle\left\langle\Omega_{2 \pi(n-1)}\right|+\cdots,
$$

where $\left|\Omega_{2 \pi n}\right\rangle$ represents the ground state of the system in a $2 \pi n$ flux background, and... represents less important contributions such as those from excited states. In fact, since we are primarily interested in fluctuations near zeroflux background, we can focus on the effect of the $n=1$ term for most of our purposes. All of these results can be made more precise by using techniques in conformal field theories such as radial quantization and the state-operator correspondence [48], but for the purpose of this work, we do not need to use that machinery.

The monopole operator is not included in Eq. (4) but, in principle, may be included as a perturbation that explicitly breaks the $U(1)_{\text {top }}$ symmetry. In the absence of the gapless Dirac fermions (or other matter fields), it is known that such a perturbation [25] will open a gap for the Maxwell photon and confine gauge charges. With gapless matter fields (like the Dirac fermions here), the effect of monopole perturbation is more subtle: At large enough $N_{f}$ (fermion flavor), the monopole becomes an irrelevant perturbation, but the lower critical $N_{f}$ is not completely known (some bounds were estimated from the F-theorem [49]).
Let us look at the monopoles in more detail. It is helpful to think about the large- $N_{f}$ limit, where gauge fluctuations are suppressed. In this case, the monopole simply creates a static $2 \pi$ magnetic flux in which the Dirac fermions move freely. The most relevant (in the renormalization group sense) monopole corresponds to the ground state of these Dirac fermions, with all negative-energy levels filled and all positive-energy levels empty. However, each Dirac cone also contributes to a zero-energy mode (guaranteed by the Atiyah-Singer index theorem) in a $2 \pi$ flux background. The filling of any of these four zero modes does not affect energetics. However, gauge invariance (i.e., vanishing of the overall gauge charge) requires that exactly half of the zero modes be filled [27]. This requirement gives a total of $C_{4}^{2}=6$ distinct (but equally relevant) monopoles, schematically written as

$$
\Phi \sim f_{i}^{\dagger} f_{j}^{\dagger} \mathcal{M}_{\text {bare }},
$$

where $f_{i}^{\dagger}$ creates a fermion in the zero mode associated with $\psi_{i}$, and $\mathcal{M}_{\text {bare }}$ creates a "bare" flux quantum without filling any zero mode. For later convenience, we define the six monopoles as

$$
\begin{aligned}
& \Phi_{1 / 2 / 3}^{\dagger}=f_{\alpha, s}^{\dagger}\left(\epsilon \tau^{1 / 2 / 3}\right)^{\alpha \beta} \epsilon^{s s^{\prime}} f_{\beta, s^{\prime}}^{\dagger} \mathcal{M}_{\text {bare }}, \\
& \Phi_{4 / 5 / 6}^{\dagger}=i f_{\alpha, s}^{\dagger}(\epsilon)^{\alpha \beta}\left(\epsilon \sigma^{1 / 2 / 3}\right)^{s s^{\prime}} f_{\beta, s^{\prime}}^{\dagger} \mathcal{M}_{\text {bare }},
\end{aligned}
$$

where we refine the label of the zero mode by valley indices $\alpha=1,2$ and spin indices $s=\uparrow, \downarrow ; \epsilon$ is the antisymmetric rank-2 tensor, which is necessary because of the anticommutation relations of $f$ operators; and $\tau, \sigma$ acts on valley or spin indices as the standard Pauli matrices formalism. The factor $i$ in the second line is necessary because the six monopoles are related by $S U(4)$ rotations of Dirac fermions (to be explained in more detail later). From our construction, it is straightforward to see that the first three monopoles are spin singlets, while the latter three monopoles are spin triplets. Both the Dirac sea and the zero modes contribute to properties of monopoles such as symmetry quantum numbers.

One can likewise define "antimonopoles" as operators inserting $-2 \pi$ flux and appropriately filling Dirac zero modes. However, it is more convenient for us to simply view such operators as the "antiparticles," or hermitian conjugates, of the monopole operators defined above.

Notice that under a $2 \pi$ flux, each zero mode behaves as a Lorentz scalar [27] (since there is no other index responsible for higher spin), in contrast to its parent Dirac fermion (originally a spinor). This makes the monopole operator also a Lorentz scalar.

\section{B. Symmetries}

We now carefully examine the global symmetries of the continuum $\mathrm{QED}_{3}$ theory. Clearly, we have the Lorentz symmetry, together with the standard charge conjugation $\mathcal{C}$, 
time reversal $\mathcal{T}$, and space reflection $\mathcal{R}_{x}$. As we have discussed already, the conservation of the gauge flux corresponds to a global $U(1)$ symmetry known as the topological $U(1)_{\text {top. }}$. The fermion flavor symmetry is naively $S U(4): \psi_{i} \rightarrow U_{i j} \psi_{j}$, where $U \in S U(4)$, but we should remember that global symmetries, properly defined, should only act on gauge-invariant local operators. Naively, one would consider fermion bilinear operators like $\bar{\psi} \sigma^{\mu} \tau^{\nu} \psi$ as the simplest gauge-invariant operators, which transform as (15-dimensional) $S U(4)$ adjoints. However, the monopole operators [defined in Eqs. (7) or (8)] transform as a sixdimensional vector under $S U(4)$, or more precisely $S O(6)=S U(4) / \mathbb{Z}_{2}$. Notice that this operator is odd under both the $S O(6)$ center $\left(-I_{6 \times 6}\right)$ and a $\pi$ rotation in $U(1)_{\text {top }}$. More generally, one can show that any local operator has to be simultaneously odd or even under the two operationsfor example, a fermion bilinear $\bar{\psi}_{i} \psi_{j}$ carries no gauge flux and is even under the $S O(6)$ center. So the proper global symmetry group should be

$$
\frac{S O(6) \times U(1)_{\text {top }}}{\mathbb{Z}_{2}}
$$

together with $\mathcal{C}, \mathcal{T}, \mathcal{R}_{x}$ and Lorentz. One can certainly consider $2 \pi$ monopoles in higher representations of $S O(6)$, but in this work, we will that the leading monopoles (with lowest scaling dimension) are the ones forming an $S O(6)$ vector - this is physically reasonable and can be justified in the large- $N_{f}$ limit.

Instead of working with the explicit definition of monopoles from Eq. (7), we simply think of the monopoles as six operators $\left\{\Phi_{1}, \ldots \Phi_{6}\right\}$ that carry unit charge under $U(1)_{\text {top }}$ and transform as a vector under $S O(6): \Phi_{i} \rightarrow$ $O_{i j} \Phi_{j}$. Likewise, we define "antimonopoles" as six operators $\left\{\Phi_{1}^{\dagger}, \ldots \Phi_{6}^{\dagger}\right\}$ that also transform as an $S O(6)$ vector but carry -1 charge under $U(1)_{\text {top }}$. The virtue of defining the monopole operators abstractly based on symmetry representations is that we can easily fix the $\mathcal{C}, \mathcal{T}, \mathcal{R}_{x}$ symmetry actions on the monopoles completely based on the group structure. Consider a "bare" time-reversal symmetry

$$
\mathcal{T}_{0}: \psi \rightarrow i \gamma_{0} \sigma^{2} \tau^{2} \psi
$$

where $\gamma$ acts on the Dirac index, $\sigma$ acts on the physical spin index, and $\tau$ acts on the "valley" index. The physical timereversal symmetry $\mathcal{T}$ (to be discussed later) is, in general, a combination of $\mathcal{T}_{0}$ and some additional $S U(4)$ rotation $U_{T}$. Now, consider the group structure of the $S O(6) \times$ $U(1)_{\text {top }} / \mathbb{Z}_{2}$ symmetry and $\mathcal{T}_{0}$. Clearly, $\mathcal{T}_{0}$ commutes with $U(1)_{\text {top }}$, which simply means that monopoles become antimonopoles. Note that $\mathcal{T}_{0}$ also commutes with $S U(4)$ rotations generated by $\left\{\sigma^{i}, \tau^{i}\right\}$, namely, the spin-valley subgroup $S O(3)_{\text {spin }} \times S O(3)_{\text {valley }}$. But for those generated by $\left\{\sigma^{i} \tau^{j}\right\}$, we have $\mathcal{T}_{0} U=U^{\dagger} \mathcal{T}_{0}$. One can then show that the only consistent implementations on the monopoles are $\mathcal{T}_{0}: \Phi \rightarrow \pm O_{T} \Phi^{\dagger}$, where

$$
O_{T}=\left(\begin{array}{cc}
I_{3 \times 3} & 0 \\
0 & -I_{3 \times 3}
\end{array}\right) .
$$

The basis is chosen so that $\Phi_{1,2,3}$ rotates under the $S O(3)$ generated by $\tau^{i}$, and $\Phi_{4,5,6}$ rotates under that by $\sigma^{i}$. Importantly, $O_{T} \in O(6)$ but not $S O(6)$. One can likewise consider a "bare" reflection

$$
\mathcal{R}_{0}: \psi(x) \rightarrow i \gamma_{1} \psi(\mathcal{R} x)
$$

Since this symmetry commutes with $S O(6)$ rotations but flips $U(1)_{\text {top }}$ charge, we have, for the monopoles, $\mathcal{R}_{0}: \Phi_{i} \rightarrow$ $\Phi_{i}^{\dagger}$ (up to a phase factor that can be absorbed through a redefinition of $\Phi)$. Finally, for the "bare" charge conjugation,

$$
\mathcal{C}_{0}: \psi \rightarrow \sigma^{2} \tau^{2} \psi^{*}
$$

We notice that it has the same commutation relation to $S O(6)$ as $\mathcal{T}_{0}$ and also flips $U(1)_{\text {top }}$ charge. Therefore, $\mathcal{C}_{0}: \Phi \rightarrow \pm O_{T} \Phi^{\dagger}$. This analysis also shows that the fermion mass operators $\bar{\psi}_{i} T_{i j} \psi_{j}$ that form an adjoint representation of $S U(4)$ [ $T$ is an $S U(4)$ generator] is indistinguishable from $i \Phi_{i}^{\dagger} A_{i j} \Phi_{j}$ in terms of symmetry quantum numbers, where $A$ is a real $6 \times 6$ antisymmetric matrix.

Clearly, the lattice spin Hamiltonians would not have the full continuum symmetry-typically, we only have spin rotation, lattice translation and rotation, reflection, and time-reversal symmetries. It was argued in Ref. [13] that the enlarged symmetry [such as $S O(6) \times U(1)_{\text {top }} / \mathbb{Z}_{2}$ ] would emerge in the IR theory since terms breaking this symmetry down to the microscopic ones are likely to be irrelevant (justified in large- $N_{f}$ analysis). In this work, we will assume that the enlarged symmetry does emerge, at least before the monopole tunneling is explicitly added to the Lagrangian.

The central question in this paper is as follows: Given a realization of a $U(1)$ Dirac spin liquid on some lattice, how do the monopoles transform under the microscopic symmetries (such as lattice translation)? Since we already know how the monopoles transform under the IR emergent symmetries [such as $S O(6) \times U(1) / \mathbb{Z}_{2}$ ], the question can be equivalently formulated as follows: How are the microscopic symmetries embedded into the enlarged symmetry group? Clearly, spin rotation can only be embedded as an $S O(3)$ subgroup of the $S O(6)$ flavor group, meaning that three of the six monopoles [ $\Phi_{4,5,6}$ from Eq. (8)] form a spin-1 vector, and the other three [ $\Phi_{1,2,3}$ from Eq. (8)] are spin singlets. Other discrete symmetries can be realized, in general, as combinations of certain $S O(6)$ rotations 
followed by a nontrivial $U(1)_{\text {top }}$ rotation, and possibly some combinations of $\mathcal{C}_{0}, \mathcal{T}_{0}, \mathcal{R}_{0}$. (Remember that the Lorentz group acts trivially on the $2 \pi$ monopoles.) In fact, in all the examples we are interested in, all those discrete symmetries commute with the spin $S O(3)$ rotation. This means that for most purposes, we can focus on the $S O(3)_{\text {spin }} \times S O(3)_{\text {valley }}$ subgroup of $S O(6)$, and the realization of the discrete symmetries should only involve $S O(3)_{\text {valley }}$ and possibly $\mathcal{C}, \mathcal{P}, \mathcal{T}$.

Many of these group elements in a symmetry realization can be fixed from the symmetry transformations of the Dirac fermions $\psi_{i}$, which are fixed by the symmetry of the mean-field ansatz in Eq. (3), under the name projective symmetry group (PSG) [5]. For example, if the symmetry operation acts on $\psi$ as $\psi \rightarrow U \psi$ with a nontrivial $U \in S U(4)$, then we know that the monopoles should also be multiplied by an $S O(6)$ matrix $O$ that corresponds to $U$. This $S O(6)$ matrix $O$ can be uniquely identified up to an overall sign, which can also be viewed as a $\pi$ rotation in $U(1)_{\text {top }}$. In practice, the $S O(6)$ flavor rotation involved in a symmetry realization is always within the $S O(3)_{\text {spin }} \times$ $S O(3)_{\text {valley }}$ subgroup. The six monopoles transform as $(1,0) \oplus(0,1)$ under this subgroup, which is the same representation of the six fermion bilinears $\left\{\bar{\psi} \sigma^{i} \psi, \bar{\psi} \tau^{i} \psi\right\}$. Therefore, to fix the $S O(6)$ rotation of the monopoles in a given symmetry realization, it is sufficient to fix that for the six masses, also known as quantum spin Hall and quantum valley Hall masses, respectively. For the examples we are interested in, this information is also reviewed in Appendix A.

Similar logic applies to operations like $\mathcal{C}, \mathcal{T}, \mathcal{R}_{x}$. The only exception is the flux symmetry $U(1)_{\text {top }}$ : There is no information regarding $U(1)_{\text {top }}$ in the PSG. Fixing the possible $U(1)_{\text {top }}$ rotations in the implementations of the microscopic discrete symmetries is the main task of this work.

The difficulty in fixing the $U(1)_{\text {top }}$ factor in a symmetry transform lies in its UV nature: Intuitively, the $U(1)_{\text {top }}$ phase factor comes from the Berry phase accumulated when a monopole moves on the lattice scale, in a nontrivial "charge background" [29,30]. This lattice-scale feature is not manifested directly in simple objects in the IR (such as the Dirac fermions). In previous studies, such phase factors were decided numerically $[28,33,50]$. In this paper, we develop several different analytical methods to calculate such phase factors.

We should emphasize here that the questions addressed in this work are kinematic (rather than dynamical) in nature; i.e., we are interested in the qualitative properties of the monopoles, such as symmetry representations, rather than quantitative properties such as scaling dimensions. We introduce, at various stages of our argument, assumptions that are only justified in certain limits (such as at large $N_{f}$ ), and importantly, although these assumptions will not be completely satisfied, they will provide a rationale for selecting an answer typically from a discrete set of possibilities. Some of the particularly important assumptions that follow from this treatment are as follows: (1) The most relevant monopole operators are those that transform as an $S O(6)$ vector and Lorentz scalar-this case is physically reasonable but justifiable only in large $N_{f}$; (2) when perturbed by an adjoint mass $\bar{\psi} \sigma^{\mu} \tau^{\nu} \psi$, the small mass limit is adiabatically connected with the large mass limit, which describes lattice scale physics and the Wannier limit (roughly speaking, this means that the adjoint mass is not only relevant but flows all the way to infinity in the IR) [51] - another physically reasonable assumption that is justified in large $N_{f}$; and (3) the $U(1)_{\text {top }}$ phase factors in the microscopic symmetry realizations are decided completely by the mean-field theory of the spinons (which is a free-fermion theory)—gauge fluctuations only modify other quantitative features (such as scaling dimensions) but not the (discrete) symmetry properties. In particular, assumption (3) may not always be valid (depending on microscopic details), but when it is not valid, the parton construction combined with the mean-field description is itself not likely to provide a reasonable starting point to describe the phase.

\section{TIME-REVERSAL, REFLECTION, AND BAND TOPOLOGY}

We now derive some general results for time-reversal and reflection symmetries that will be applicable in all the systems we are interested in. Essentially, with the help of the exact $S O(3)$ spin rotation symmetry, the $U(1)_{\text {top }}$ phase factors associated with time reversal and reflection can be uniquely fixed. We remark that this phase factor cannot be determined numerically.

Since the $U(1)_{\text {top }}$ phase factor comes from UV physics, we can deform the $\mathrm{QED}_{3}$ theory with a fermion mass to make the theory IR trivial so that we can focus on the UV part. Consider perturbing with a mass term

$$
\Delta \mathcal{L}=m \bar{\psi}_{i} T_{i j} \psi_{j}
$$

where $T_{i j}$ is chosen to be an $S U(4)$ generator without loss of generality. The fermions are now gapped, and there is a pure Maxwell $U(1)$ gauge theory left in the IR. The zero modes associated with the monopoles are lifted (according to the signs of the fermion masses), lifting the sixfold degeneracy of the monopole completely so that there is only one gapless monopole left in the Maxwell theory. The identity of the surviving monopole is fixed again by symmetry. The mass term breaks the flavor symmetry from $S O(6)$ to $S O(4) \times S O(2)$. If we probe the theory with an $S O(4) \times S O(2)$ gauge field $\mathcal{A}^{S O(4)}+A^{S O(2)}$, the massive fermions generate a topological term 


$$
\mathcal{L}_{\text {top }}=\frac{\operatorname{sgn}(m)}{2 \pi} A^{S O(2)} d a
$$

This means that the monopole (now unique) carries \pm 1 charge under the $S O(2)$ generated by $T$ in Eq. (14) and is a singlet under the remaining $S O(4)$ - this uniquely fixes the identity of the monopole among the six degenerate ones in the gapless phase. The argument will also be useful for deciding the nature of the symmetry-breaking phase when a mass perturbation is turned on since the monopole will eventually spontaneously condense in the Maxwell theory, possibly breaking further symmetries [such as the $S O(2)$ here].

Now, as long as the relevant symmetry, let us call it $g$, is not broken by the mass perturbation, the $U(1)_{\text {top }}$ phase factor $U_{\text {top }}^{g}$ associated with the implementation of $g$ will not be affected. We are thus left with the simpler problem of finding the Berry phase of a nondegenerate monopole moving in an insulating (gapped) charge background, which is essentially determined by the topology of the insulator. Since the other monopoles are related to this monopole by some $S O(6)$ flavor rotations, their symmetry transformations are fixed once we obtain the transformation of this particular monopole.

It turns out to be particularly useful, for all the examples to be considered in this work, to consider a "quantum spin Hall" (QSH) mass perturbation

$$
\Delta \mathcal{L}_{\mathrm{QSH}}=m \bar{\psi} \sigma^{3} \psi
$$

where $\sigma^{3}$ acts in the spin but not the valley index. This term breaks the spin $S O(3)$ rotation down to $S O(2)$ and generates a mutual spin-charge Hall response as in Eq. (15), so the low-energy monopole operator transforms as $S_{z}=\operatorname{sgn}(m)$-in our notation, this monopole is denoted as $\Phi_{4} \pm i \Phi_{5}$. Crucially, this term leaves all other discrete symmetries unbroken-except for reflection symmetries $\mathcal{R}$, but it is still a symmetry when combined with a spin-flip operation $\mathcal{R}^{\prime}=\sigma^{2} \mathcal{R}$. Since the $U(1)_{\text {top }}$ factors can be nontrivial only for lattice symmetries and time reversal, they can all be determined by considering monopole quantum numbers in this QSH insulator. For example, it is well known that the QSH insulator is also a $\mathbb{Z}_{2}$ topological insulator [52], protected by the Kramers time-reversal symmetry $\mathcal{T}: f \rightarrow i \sigma^{2} f$. This case fixes the transformation of the monopole under time reversal to be

$$
\mathcal{T}: \mathcal{M} \rightarrow-\mathcal{M}^{\dagger}
$$

where the nontriviality of the topological insulator is manifested in the minus sign. This result can be seen most easily through the edge state of the QHE insulator,

$$
H_{\text {edge }}=\int d x \chi^{\dagger} i \sigma^{2} \partial_{x} \chi,
$$

where $\chi$ is a two-component Dirac fermion in $(1+1) d$, with time reversal acting as $\chi \rightarrow i \sigma^{2} \chi$ that forbids any mass term. In the QSH state, a monopole tunneling event (i.e., an adiabatic process that changes the flux by $2 \pi$ ) will transfer one left-moving fermion into a right-moving one, which is nothing but the physics of axial anomaly. This result leads to the operator identification on the edge $\mathcal{M} \sim \chi^{\dagger}\left(\sigma^{1}+i \sigma^{3}\right) \chi$, from which Eq. (17) follows.

Reflection symmetry can be discussed in a similar manner. If the reflection does not involve charge conjugation, the monopole simply transforms as

$$
\mathcal{R}_{x}: \mathcal{M} \rightarrow e^{i \theta_{\mathcal{R}}} \mathcal{M}^{\dagger},
$$

where the overall phase factor can change by a redefinition of $\mathcal{M}$ and is therefore physically meaningless-unless there is more than one reflection axis, in which case the relative phases in the transforms become meaningful. Charge conjugation symmetry (if it exists) alone is not meaningful for the same reason.

It becomes more meaningful, and turns out to be also much simpler, when a reflection involves an extra charge conjugation operation, denoted as $\mathcal{C} \mathcal{R}$. Under this symmetry, the monopole is mapped to itself, possibly with a sign

$$
\mathcal{C R}: \mathcal{M} \rightarrow \pm \mathcal{M}
$$

where we have assumed that $(\mathcal{C R})^{2}=1$ on local operators. The two different signs in the above transformation are physically distinct and represent different topology of the underlying insulators.

In a quantum spin Hall insulator, it turns out to be particularly simple to tell if the insulator is also nontrivial under an additional $\mathcal{C R}$ : It is trivial if $(\mathcal{C R})^{2} \psi=-\psi$ and nontrivial if $(\mathcal{C R})^{2} \psi=+\psi$ [which then leads to the nontrivial sign in Eq. (20)]. In particular, the "bare" $\mathcal{C R}$ symmetry defined in Eqs. (13) and (12) squares to one and therefore has nontrivial transformation.

The easiest way to understand the above statement is to consider the edge state, Eq. (18), that preserves charge, spin- $S_{z}$, and $\mathcal{C} \mathcal{R}$ symmetry. There are two different ways to implement a $\mathcal{C R}$ symmetry: $\mathcal{C} \mathcal{R}_{+}: \chi(x) \rightarrow \chi^{\dagger}(-x)$ or $\mathcal{C} \mathcal{R}_{-}$: $\chi(x) \rightarrow \sigma^{2} \chi^{\dagger}(-x)$, where $\left(\mathcal{C} \mathcal{R}_{+}\right)^{2}=1$ and $\left(\mathcal{C} \mathcal{R}_{-}\right)^{2}=-1$ on the fermions. It is easy to see that $\mathcal{C} \mathcal{R}_{+}$forbids a Dirac mass term, making the insulator also nontrivial under $\mathcal{C} \mathcal{R}_{+}$, while $\mathcal{C} \mathcal{R}_{-}$does not forbid any mass term and is therefore trivial. The monopole transformation under $\mathcal{C} \mathcal{R}$ in Eq. (20) can be obtained through the operator identification $\mathcal{M} \sim \chi^{\dagger}\left(\sigma^{1}+i \sigma^{3}\right) \chi$. This result is perhaps natural when we think of $\mathcal{C R}$ as obtained from Wick-rotating timereversal symmetry $\mathcal{T}$ using the $\mathcal{C} \mathcal{R} \mathcal{T}$ theorem [53], where $\mathcal{T}^{2}=(-1)^{F}$ rotates to $(\mathcal{C R})^{2}=+1$.

It is now natural to ask what would happen if we had a "quantum valley Hall" mass $\bar{\psi} \tau \tau^{i} \psi$ instead. Now, since the full 
spin $S O(3)$ is unbroken, the insulator cannot have the band topology of topological insulators (under either $\mathcal{T}$ or $\mathcal{C R}$ ) this is simply the famous statement that a topological insulator requires spin-orbit coupling. Therefore, time reversal and reflection (or $\mathcal{C R}$ ) should act trivially on spin-singlet monopoles selected by the valley Hall masses [54].

To summarize, the two symmetries $\mathcal{T}_{0}$ and $\mathcal{C} \mathcal{R}_{0}$ defined in Eqs. (10), (12), and (13) acts on the six monopoles as

$$
\begin{array}{r}
\mathcal{T}_{0}:\left(\begin{array}{l}
\Phi_{1,2,3} \\
\Phi_{4,5,6}
\end{array}\right) \rightarrow\left(\begin{array}{c}
\Phi_{1,2,3}^{\dagger} \\
-\Phi_{4,5,6}^{\dagger}
\end{array}\right), \\
\mathcal{C R}_{0}:\left(\begin{array}{l}
\Phi_{1,2,3} \\
\Phi_{4,5,6}
\end{array}\right) \rightarrow\left(\begin{array}{c}
\Phi_{1,2,3} \\
-\Phi_{4,5,6}
\end{array}\right) .
\end{array}
$$

The physical time reversal and reflection may further involve additional $S O(6)$ rotations or charge conjugations, which can be included straightforwardly.

Next, we turn to the more complicated symmetries including lattice translation and rotations. We first discuss the simpler cases on bipartite lattices.

\section{MONOPOLE QUANTUM NUMBERS I: BIPARTITE LATTICES}

\section{A. Monopole quantum numbers constrained by $\mathbf{Q C D}_{\mathbf{3}}$}

On bipartite lattices, at least for the examples considered in this work, we can always continuously tune the meanfield Hamiltonian, Eq. (3), without breaking any symmetry or changing the low-energy Dirac dispersion, to reach a point with particle-hole symmetry:

$$
\mathcal{C}: f_{i, \alpha} \rightarrow \eta_{i} i \sigma_{\alpha \beta}^{2} f_{i, \beta}^{\dagger},\left(\eta_{i}=\left\{\begin{array}{lc}
1 & i \in A \text { sublattice } \\
-1 & i \in B \text { sublattice }
\end{array}\right),\right.
$$

where one partitions the lattice sites into two sublattices $A$, $B$ such that neighboring sites belong to different sublattices. This theory will then have a larger gauge symmetry of $S U(2)_{g}$, with the detailed gauge transform acting as

$$
\left(f_{i, \uparrow}, f_{i, \downarrow}^{\dagger}\right)^{T} \rightarrow \begin{cases}G_{i}\left(f_{i, \uparrow}, f_{i, \downarrow}^{\dagger}\right)^{T} & i \in A \text { sublattice } \\ \sigma^{3} G_{i} \sigma^{3}\left(f_{i, \uparrow}, f_{i, \downarrow}^{\dagger}\right)^{T} & i \in B \text { sublattice }\end{cases}
$$

where $G_{i}$ is an $S U(2)$ matrix generated by Pauli matrices $\sigma^{1,2,3}$. The low-energy theory again has four Dirac cones, with two valleys. For more details, see, e.g., Ref. [5]. The continuum field theory of such a state, described by an $S U(2)$ gauge field coupled to four Dirac cones, is also known as $\mathrm{QCD}_{3}$ with $N_{f}=2$. The Lagrangian is given by

$$
\mathcal{L}_{\mathrm{QCD}}=\sum_{i=1}^{2} \bar{\chi}_{i}\left(i \not \partial+\not \phi^{S U(2)}\right) \chi_{i} .
$$

At the lattice scale, the $S U(2)$ gauge symmetry can be Higgsed down to $U(1)$ by reinstating the particle-hole symmetry-breaking hopping (which could be weak), and our familiar $U(1)$ Dirac spin liquid will be recovered at low energy. However, it turns out to be very useful to consider an intermediate theory in which the $S U(2)$ gauge symmetry is Higgsed down to $U(1)$, but the particle-hole symmetry survives as a global $\mathbb{Z}_{2}^{C}$ symmetry. At low energy, this theory also flows to $\mathrm{QED}_{3}$ but with an extra $\mathbb{Z}_{2}^{C}$ symmetry compared to the $U(1)$ Dirac spin liquid. In fact, this theory does not faithfully represent a lattice spin system due to the extra $\mathbb{Z}_{2}^{C}$. However, it is a perfectly well-defined lattice gauge theory and can represent a lattice spin system if the spin-rotation symmetry is enlarged from $S O(3)$ to $O(3) \sim S O(3) \times \mathbb{Z}_{2}^{C}$. In the continuum theory, this intermediate $\mathrm{QED}_{3}$ can be obtained from the $\mathrm{QCD}_{3}$ theory by condensing a Higgs field that carries spin 1 of the gauge $S U(2)$ and is even under $\mathbb{Z}_{2}^{C}$. This Higgs condensation does not break any global symmetry of the $\mathrm{QCD}_{3}$, and the lowenergy Dirac dispersion is not affected. We can then safely view the continuum $\mathrm{QCD}_{3}$ field theory (which is free in the $\mathrm{UV}$ ), instead of the original lattice theory, as the UV completion of the intermediate $\mathrm{QED}_{3}$ theory. As we shall see below, the virtue of this alternative UV completion is that the $\mathrm{QCD}_{3}$ theory is much easier to understand than the full lattice theory.

The QCD theory has the standard Lorentz and $\mathcal{T}, \mathcal{R}_{x}$ symmetries. It may not necessarily flow to a conformal fixed point [56], but this should not be important for our discussion since we are not interested in the ultimate IR fate of this theory. The flavor symmetry of $\mathrm{QCD}_{3}$ at $N_{f}=2$ is $S O(5)$, which acts on the fermions as the simplectic group $S p(4)$. Crucially, there is no additional topological symmetry since the flux of the $S U(2)$ gauge field is not conserved.

Now, we notice that the implementation of the microscopic (continuous or discrete) symmetries in the $\mathrm{QCD}_{3}$ theory is completely fixed by the symmetry transform of the Dirac fermions $\chi$, due to the absence of any gauge flux conservation. For example, a nontrivial $S p(4)$ transform on $\chi$ maps to a unique $S O(5)$ transform on gauge-invariant operators such as fermion bilinears [57]. The bottom line is that we know completely how the microscopic symmetries are embedded into the symmetries of the continuum $\mathrm{QCD}_{3}$ field theory. Once we reach the QCD description, the exact nature of these symmetries at the lattice scale is no longer important-we simply view them as part of the $S O(5) \times$ Lorentz $\times \mathcal{T}, \mathcal{R}_{x}$ symmetry. Now, we Higgs the $S U(2)$ gauge symmetry down to $U(1)$, and far below the Higgs scale, we obtain the intermediate $\mathrm{QED}_{3}$ theory, which has a larger emergent symmetry, including the $S O(6) \times U(1) / \mathbb{Z}_{2}$ and $\mathbb{Z}_{2}^{C}$ symmetries. 
We now show that there is a unique way to embed the symmetries of QCD $[S O(5)$ and $\mathcal{R}, \mathcal{T}]$ into the symmetries of QED. This method will, in turn, fix the embedding of the microscopic symmetries into the symmetries of QED. First, it is obvious that there is a unique way to embed the continuous $S O(5)$ symmetry of QCD to $S O(6) \times U(1) / \mathbb{Z}_{2}$ of QED, up to reordering of operators: Five of the six monopoles should transform as an $S O(5)$ vector, and the remaining one (call it $\Phi_{\text {trivial }}$ ) should be an $S O(5)$ singlet, or $6=1 \oplus 5$. Since the microscopic spin rotation symmetry $S O(3)_{\text {spin }}$ must be part of the $S O(5)$, the $S O(5)$ singlet monopole $\Phi_{\text {trivial }}$ must also be a spin singlet; i.e., it is a combination of $\Phi_{1,2,3}$. Crucially, there is no nontrivial $U(1)_{\text {top }}$ phase factor involved in the realization of the $S O(5)$ symmetry on the monopoles.

Now, consider the bare time reversal and reflection conjugation defined in Eqs. (10), (12), and (13). As we argued before, they act on the monopoles as Eq. (21). This result immediately implies that the $S O(5)$ singlet monopole $\Phi_{\text {trivial }}$ also transforms trivially under $\mathcal{T}_{0}$ and $\mathcal{C R}_{0}$. The physical time-reversal and reflection symmetry may involve a further $S O(5)$ flavor rotation, but this will not affect $\Phi_{\text {trivial }}$ since it is an $S O(5)$ singlet. For the chargeconjugation symmetry $\mathcal{C}$, we expect $\Phi_{\text {trivial }} \rightarrow e^{i \theta} \Phi_{\text {trivial }}^{\dagger}$ for some phase factor $e^{i \theta}$ since $\mathcal{C}$ cannot mix the $S O(5)$ singlet with the $S O(5)$ vector, and the phase can be chosen to be trivial by redefining the monopole operators. In this case, $\operatorname{Re} \Phi_{\text {trivial }} \equiv\left(\Phi_{\text {trivial }}+\Phi_{\text {tivial }}^{\dagger}\right) / 2$ is trivial under $\mathcal{C}$ (in later examples, sometimes the opposite convention is chosen). We then conclude that $\operatorname{Re} \Phi_{\text {trivial }}$ is trivial under all microscopic symmetries in the intermediate $\mathrm{QED}_{3}$ theory.

We now consider the actual $U(1)$ Dirac spin liquid of interest to us. This spin liquid can be obtained from the intermediate $\mathrm{QED}_{3}$ by explicitly breaking the $\mathcal{C}$ symmetry. It is also possible, as we shall see on the square lattice, that some other symmetries such as translation $T_{1,2}$ and time reversal $\mathcal{T}$ are also broken, but the combinations $T_{1,2} \mathcal{C}$ and $\mathcal{T C}$ are preserved. In any case, the symmetry-breaking term does not change the low-energy Dirac dispersion (except velocity anisotropy) and is expected to be irrelevant. Therefore, we do not expect any change in monopole symmetry quantum numbers - as long as the symmetries are still unbroken. In particular, the trivial monopole $\Phi_{\text {trivial }}$, or at least $\operatorname{Re} \Phi_{\text {trivial }} \equiv\left(\Phi_{\text {trivial }}+\Phi_{\text {trivial }}^{\dagger}\right) / 2$, should still transform trivially under all global symmetries.

In summary, on bipartite lattices, there is always one monopole operator (at least the real or imaginary part of it) that transforms trivially under all microscopic symmetries. The reasoning is summarized in Fig. 2.

Although we have emphasized the bipartiteness of the lattices in our argument, the discussion above shows that what really matters is whether the $U(1)$ spin-liquid meanfield ansatz could be upgraded to an $S U(2)$ gauge theory. One could certainly consider the mean-field ansatz on

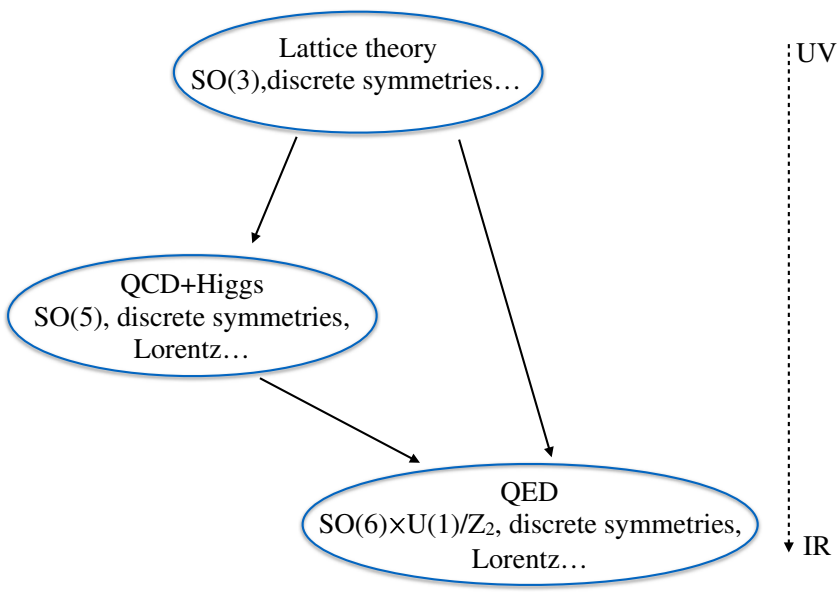

FIG. 2. For the Dirac spin liquid on bipartite lattices (honeycomb and square), we can view the continuum $\mathrm{QCD}_{3}+$ Higgs field theory (instead of the original lattice theory) as the UV completion of the $\mathrm{QED}_{3}$ theory. The implementation of microscopic symmetries (at the lattice scale) in the QCD theory can be fixed by PSG analysis, while the implementation of QCD symmetries in the QED theory is also uniquely fixed by field theory analysis. This uniquely fixes the implementation of microscopic symmetries in the QED [ $U(1)$ Dirac spin-liquid] theory.

bipartite lattices that are "intrinsically nonbipartite," meaning they cannot be adiabatically tuned to have an $S U(2)$ gauge structure. One could also consider the ansatz on nonbipartite lattices that are compatible with $S U(2)$ gauge structure, for example, by making all the hoppings imaginary (giving up time-reversal symmetry, although this would typically induce a gap). Our dichotomy on bipartiteness should be applied with care in those scenarios.

The above argument also completely fixes all the monopole quantum numbers on bipartite lattices. We now look at honeycomb and square lattices in detail.

\section{Honeycomb lattice}

The uniform hopping mean-field ansatz on the honeycomb lattice gives the $N_{f}=4 \mathrm{QED}_{3}$ low-energy theory. The Dirac points stay at momenta $\mathbf{K}=[(2 \pi / 3)$, $(2 \pi / 3)], \mathbf{K}^{\prime}=-\mathbf{K}$. Under an appropriate basis, the lowenergy effective Hamiltonian takes the standard Dirac form. The physical symmetries act as

$$
\begin{aligned}
T_{1 / 2}: \psi & \rightarrow e^{-i(2 \pi / 3) \tau^{3}} \psi, \quad C_{6}: \psi \rightarrow-i e^{-i(\pi / 6) \mu^{3}} \tau^{1} e^{-i(2 \pi / 3) \tau^{3}} \psi, \\
R_{x}: \psi & \rightarrow-\mu^{2} \tau^{2} \psi, \quad R_{y}: \psi \rightarrow \mu^{1} \tau^{3} \psi, \\
\mathcal{T}: \psi & \rightarrow-i \sigma^{2} \mu^{2} \tau^{2} \psi, \quad \mathcal{C}: \psi \rightarrow i \mu^{1} \tau^{1} \sigma^{2} \psi^{*}
\end{aligned}
$$

where $\mu^{i}$ are Pauli matrices acting on the Dirac spinor index, $T_{1 / 2}$ is the translation along two basis vectors with a $2 \pi / 3$ angle between them, $C_{6}$ is the $\pi / 3$ rotation around the center of a honeycomb plaquette, and $R_{x / y}$ denotes the 
reflection perpendicular to the direction of the unit cell or the axis perpendicular to the unit cell direction, respectively.

As an illustrative example, let us consider the $C_{6}$ rotation (the most nontrivial symmetry here). In general, the six monopoles could transform as $\Phi_{i} \rightarrow e^{i \theta_{C_{c}}} O_{i j} \Phi_{j}$, with $e^{i \theta_{C_{6}}}$ an overall phase and the $S O(6)$ matrix $O$ given by

$$
O=\left(\begin{array}{cccccc}
-\cos (2 \pi / 3) & \sin (2 \pi / 3) & 0 & 0 & 0 & 0 \\
\sin (2 \pi / 3) & \cos (2 \pi / 3) & 0 & 0 & 0 & 0 \\
0 & 0 & 1 & 0 & 0 & 0 \\
0 & 0 & 0 & -1 & 0 & 0 \\
0 & 0 & 0 & 0 & -1 & 0 \\
0 & 0 & 0 & 0 & 0 & -1
\end{array}\right)
$$

The form of the matrix $O_{i j}$ is fixed by the symmetry transforms of the Dirac fermions $C_{6}: \psi \rightarrow \tau^{1} \exp \left[-i(2 \pi / 3) \tau^{3}\right] \psi$, up to an overall sign that can be absorbed into the overall phase factor. Our argument implies that the phase factor $e^{i \theta_{C_{6}}}$ should be chosen so that the total transform takes the form

$$
\left(\begin{array}{cc}
1 & 0 \\
0 & S O(5)
\end{array}\right)
$$

with the trivial monopole being a spin singlet. Since $O$ already takes the above form (with $\Phi_{3}$ being a trivial singlet), the additional $U(1)_{\text {top }}$ phase factor must be trivial. Focusing on the spin-triplet monopoles $\left(\Phi_{4,5,6}\right)$, this reproduces the result previously obtained through numerical calculations [35].

The other symmetry actions can be determined using the same logic. The results are tabulated in Table I.

An important conclusion is that the operator $\Phi_{3}+\Phi_{3}^{\dagger}$ transforms trivially under all physical symmetries and is therefore allowed as a perturbation to the $\mathrm{QED}_{3}$ theory.

\section{Square lattice}

We consider the staggered flux state on the square lattice, with lattice hopping amplitudes (in a certain gauge)

$$
t_{i, i+\hat{y}}=\exp \left[i(-1)^{x+y} \theta / 2\right] t, \quad t_{i, i+\hat{x}}=(-1)^{y} t .
$$

The staggered flux state can be continuously tuned (without changing the low-energy Dirac dispersion) to have $\theta=0$, also known as the $\pi$-flux state. The $\pi$-flux state has all the symmetries of the staggered flux state, together with an additional particle-hole symmetry. In fact, the $\pi$-flux state has an $S U(2)$ gauge symmetry, and the unit cell contains two sites (sublattices $A, B$ ) with a vertical link. As far as the monopole quantum number is concerned, there is no distinction between the two except for the particle-hole symmetry, which does not exist in the staggered flux state. We therefore calculate the monopole quantum number in the $\pi$-flux state, which is simpler.

There are two gapless points in the reduced Brillouin zone at $\mathbf{Q}=(\pi / \mathbf{2}, \pi), \mathbf{Q}^{\prime}=-\mathbf{Q}$. The low-energy theory takes the standard Dirac form in an appropriate basis.

In the $\pi$-flux phase, we can write the (projective) symmetry realizations on the Dirac fermions as

$T_{1}: \psi \rightarrow i \tau^{3} \psi, \quad T_{2}: \psi \rightarrow i \tau^{1} \psi$,

$R_{x}: \psi \rightarrow \mu^{3} \tau^{3} \psi, \quad C_{4}: \psi \rightarrow e^{i(\pi / 4) \mu^{1}} e^{-i(\pi / 4) \tau^{2}} \psi$,

$\mathcal{T}: \psi \rightarrow i \mu^{2} \sigma^{2} \tau^{2} \psi$

together with a particle-hole symmetry:

$$
\mathcal{C}: \psi \rightarrow i \mu^{3} \sigma^{2} \psi^{*}
$$

where $\mu^{i}$ are Pauli matrices acting on the Dirac spinor index, and $C_{4}$ means a fourfold rotation around a lattice site. If we turn on a nonzero $\theta$ in Eq. (28) and convert the state to the staggered flux phase, $\mathcal{C}, T_{1,2}, C_{4}, \mathcal{T}$ will be broken, but $\mathcal{C} T_{1,2}, \mathcal{C} C_{4}, \mathcal{C T}$ will be preserved and provide the realizations of the physical symmetries.

The monopole quantum numbers can now be deduced using the argument outlined above. The results are tabulated in Table II. To be concrete, we start with the $\pi$-flux state (first four rows in Table II). The $C_{4}$ operation, based on its action on the Dirac fermions, should act on the monopoles as $C_{4}: \Phi_{i} \rightarrow e^{i \theta_{C_{4}}} O_{i j}^{C_{4}} \Phi_{j}$, where

TABLE I. The transformation of monopoles on the honeycomb lattice. Note that $T_{1 / 2}$ is the translation along two basic lattice vectors, $R_{x}$ is the reflection perpendicular to the axis defined by a unit cell (shown in Fig. 6), and $C_{6}$ is the sixfold rotation around the center of a plaquette. There is a trivial monopole, i.e., the third monopole $\Phi_{3}$.

\begin{tabular}{lccccc}
\hline \hline & $T_{1}$ & $T_{2}$ & $R_{x}$ & $C_{6}$ & $\mathcal{T}$ \\
\hline$\Phi_{1}^{\dagger}$ & $\cos (2 \pi / 3) \Phi_{1}^{\dagger}-\sin (2 \pi / 3) \Phi_{2}^{\dagger}$ & $\Phi_{1}$ & $-\cos (2 \pi / 3) \Phi_{1}^{\dagger}+\sin (2 \pi / 3) \Phi_{2}^{\dagger}$ & $\Phi_{1}$ \\
$\Phi_{2}^{\dagger}$ & $\cos (2 \pi / 3) \Phi_{2}^{\dagger}+\sin (2 \pi / 3) \Phi_{1}^{\dagger}$ & $-\Phi_{2}$ & $\cos (2 \pi / 3) \Phi_{2}^{\dagger}+\sin (2 \pi / 3) \Phi_{1}^{\dagger}$ & $\Phi_{2}$ \\
$\Phi_{3}^{\dagger}$ & $\Phi_{3}^{\dagger}$ & $\Phi_{3}^{\dagger}$ & $\Phi_{3}$ & $\Phi_{3}^{\dagger}$ & $\Phi_{3}$ \\
$\Phi_{4 / 5 / 6}^{\dagger}$ & $\Phi_{4 / 5 / 6}^{\dagger}$ & $\Phi_{4 / 5 / 6}^{\dagger}$ & $-\Phi_{4 / 5 / 6}$ & $-\Phi_{4 / 5 / 6}^{\dagger}$ & $-\Phi_{4 / 5 / 6}$ \\
\hline \hline
\end{tabular}


TABLE II. The transformation of monopoles on the square lattice. Here, $T_{1 / 2}$ is the translation along two basic lattice vectors, $R_{x}$ is the reflection perpendicular to the horizontal axis, and $C_{4}$ is the fourfold rotation around the site (shown in Fig. 6). There is a trivial monopole by our definition, i.e., the second monopole $\Phi_{2}$. The first four rows and last four rows of the monopole transformation correspond to the $\pi$-flux state and staggered-flux state, respectively, which differ by a charge conjugation for $T_{1 / 2}, C_{4}$, and $\mathcal{T}$. The last four rows align with the results of $M$ transformations of Ref. [33] after making the identification $\quad \Phi_{1 / 3}=M_{3 / 2}, \quad \Phi_{2}=i M_{1}, \quad \Phi_{4} \mp i \Phi_{5}=M_{4 / 6}$, $\Phi_{6}=M_{5}$, where $M^{\dagger}$ 's are the "monopole operators" defined in Ref. [33]. We emphasize that the " $\pi$-flux state" with $U(1)$ gauge symmetry does not actually represent a spin system and should be viewed as an intermediate state for our purpose.

\begin{tabular}{lcccccc}
\hline \hline & $T_{1}$ & $T_{2}$ & $R_{x}$ & $C_{4}$ & $\mathcal{C}$ & $\mathcal{T}$ \\
\hline$\Phi_{1}^{\dagger}$ & + & - & $-\Phi_{1}$ & $-\Phi_{3}^{\dagger}$ & $\Phi_{1}$ & $\Phi_{1}$ \\
$\Phi_{2}^{\dagger}$ & + & + & $-\Phi_{2}$ & $\Phi_{2}^{\dagger}$ & $-\Phi_{2}$ & $\Phi_{2}$ \\
$\Phi_{3}^{\dagger}$ & - & + & $\Phi_{3}$ & $\Phi_{1}^{\dagger}$ & $\Phi_{3}$ & $\Phi_{3}$ \\
$\Phi_{4 / 5 / 6}^{\dagger}$ & - & - & $\Phi_{4 / 5 / 6}$ & $\Phi_{4 / 5 / 6}$ & $\Phi_{4 / 5 / 6}$ & $-\Phi_{4 / 5 / 6}$ \\
$\Phi_{1}^{\dagger}$ & $\Phi_{1}$ & $-\Phi_{1}$ & $-\Phi_{1}$ & $-\Phi_{3}$ & & $\Phi_{1}^{\dagger}$ \\
$\Phi_{2}^{\dagger}$ & $-\Phi_{2}$ & $-\Phi_{2}$ & $-\Phi_{2}$ & $-\Phi_{2}$ & & $-\Phi_{2}^{\dagger}$ \\
$\Phi_{3}^{\dagger}$ & $-\Phi_{3}$ & $\Phi_{3}$ & $\Phi_{3}$ & $\Phi_{1}$ & & $\Phi_{3}^{\dagger}$ \\
$\Phi_{4 / 5 / 6}^{\dagger}$ & $-\Phi_{4 / 5 / 6}$ & $-\Phi_{4 / 5 / 6}$ & $\Phi_{4 / 5 / 6}$ & $\Phi_{4 / 5 / 6}$ & & $-\Phi_{4 / 5 / 6}^{\dagger}$ \\
\hline \hline
\end{tabular}

$$
O^{C_{4}}=\left(\begin{array}{cccccc}
0 & 0 & -1 & 0 & 0 & 0 \\
0 & 1 & 0 & 0 & 0 & 0 \\
1 & 0 & 0 & 0 & 0 & 0 \\
0 & 0 & 0 & 1 & 0 & 0 \\
0 & 0 & 0 & 0 & 1 & 0 \\
0 & 0 & 0 & 0 & 0 & 1
\end{array}\right) .
$$

Based on the argument outlined before, we should have $e^{i \theta_{C_{4}}}=1$, and $\Phi_{2}$ is the $S O(5)$ singlet monopole. This result, in turn, fixes the actions of $T_{1,2}$ on monopoles. For example, the action of $T_{1}$ on the Dirac fermions requires that $T_{1}: \Phi_{i} \rightarrow e^{i \theta_{T_{1}}} O_{i j}^{T_{1}} \Phi_{j}$, where

$$
O^{T_{1}}=\left(\begin{array}{cccccc}
-1 & 0 & 0 & 0 & 0 & 0 \\
0 & -1 & 0 & 0 & 0 & 0 \\
0 & 0 & 1 & 0 & 0 & 0 \\
0 & 0 & 0 & 1 & 0 & 0 \\
0 & 0 & 0 & 0 & 1 & 0 \\
0 & 0 & 0 & 0 & 0 & 1
\end{array}\right) .
$$

Since $\Phi_{2}$ should be a singlet under flavor symmetries, we must have $e^{i \theta_{T_{1}}=-1}$, which gives the transformation tabulated in Table II.
The combined symmetry action $\mathcal{C R}_{x}$, as we discussed before, can act on the monopoles with potentially nontrivial Berry phases. For this purpose, we can view this symmetry as a combination of $\mathcal{C} \mathcal{R}_{0}$ [as defined in Eqs. (12) and (13)] and a flavor rotation $\psi \rightarrow i \tau^{1} \psi$, followed by a Lorentz rotation, which does not affect the scalar monopoles. The $\mathcal{C R}_{0}$ transforms the monopoles as Eq. (21), and the flavor rotation is essentially the $T_{2}$ transformation. Therefore, under $\mathcal{C} \mathcal{R}_{x}$, we should have $\Phi_{1} \rightarrow-\Phi_{1}$ and $\Phi_{i} \rightarrow \Phi_{i}$ for $i \neq 1$. For $\mathcal{R}_{x}$ and $\mathcal{C}$ separately, the overall phase depends on the definition of the monopoles as we discussed, but the relative transformation between the six monopoles is still meaningful. Since $\mathcal{R}_{x}$ involves a flavor rotation, it should act on the monopoles as shown in Table II up to an overall phase that we fix to be trivial. The transformation of $\mathcal{C}$ then follows immediately. Finally, the transformation of $\mathcal{T}$ on the monopoles is simply given by Eq. (21).

Now, we proceed to consider the staggered-flux state [the actual $U(1)$ Dirac spin liquid]. The only difference now is that $\mathcal{C}$ is no longer a symmetry, and the action of $T_{1,2}, C_{4}, \mathcal{T}$ should be combined with $\mathcal{C}$. The results are tabulated in Table II in the last four rows. Again, we see that there is a trivial monopole $\operatorname{Im} \Phi_{2}$ that can be added as a perturbation.

\section{B. Another approach: Lattice symmetries generated by $\mathcal{C R}$}

There is another trick to obtain the lattice-symmetry quantum number for the monopoles on bipartite lattices, thanks to the extra $\mathcal{C}$ symmetry. The key is to realize that rotation and translation symmetries can all be generated by repeatedly applying $\mathcal{C} \mathcal{R}$ symmetries with respect to different reflection axes (a simple $\mathcal{R}$ will not work since the overall phase is not well defined). We will show below that this approach gives results identical to those obtained in Sec. IVA, which gives us more confidence since the two approaches appear to be very different from each other.

Following Sec. III, we calculate the monopole lattice quantum numbers when the fermions form a quantum spin Hall insulator (which preserves all lattice symmetries). Recall that in the quantum spin Hall phase, $\mathcal{C R}: \mathcal{M} \rightarrow$ $\pm \mathcal{M}$ if $\mathcal{C} \mathcal{R}^{2}=\mp 1$ on the fermions. This gives us the quantum numbers of a particular monopole (say, $\Phi_{4}+i \Phi_{5}$ ), and those of other monopoles can be fixed by the $S O(6)$ flavor symmetry. Therefore, our task below is to show that the quantum numbers of $\Phi_{4}+i \Phi_{5}$ calculated in the quantum spin Hall phase agree with those tabulated in Tables I and II.

\section{Honeycomb lattice}

Here, we find $\left(\mathcal{C} R_{x / y}\right)^{2}=-1(1)$ when acting on fermions (see Fig. 3). This result means that (from Sec. III) the spintriplet monopoles, $\left(\Phi_{4,5,6}\right)$ stays invariant or gets a minus sign under $\mathcal{C} R_{x}, \mathcal{C} R_{y}$, respectively. All other space symmetries are generated from reflections, and we get the transformation 


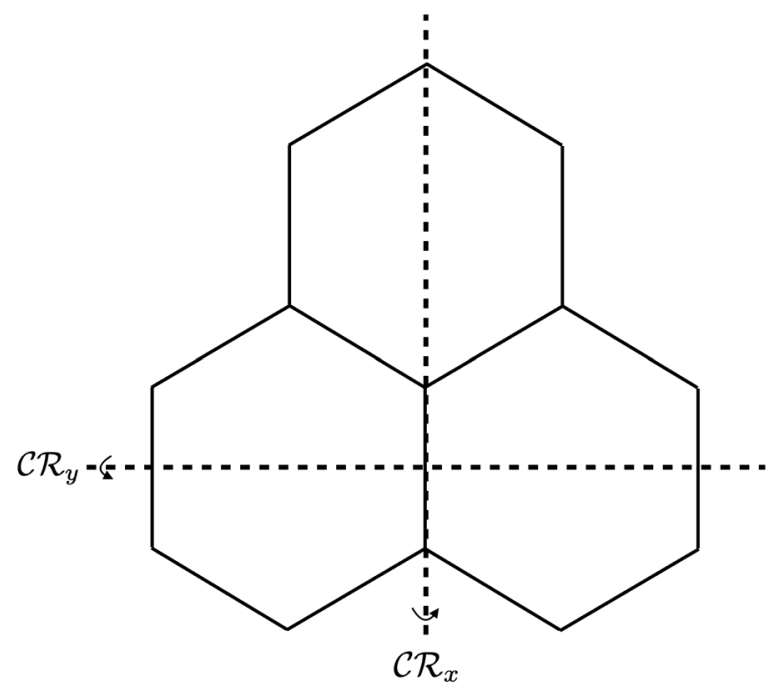

FIG. 3. Reflection axis to be considered in the main text.

results in Table I. For example, the $C_{6}$ operation can be obtained by two different $\mathcal{C} \mathcal{R}$ reflections in succession, and we immediately see that $C_{6}: \Phi_{4,5,6} \rightarrow \Phi_{4,5,6}$. Likewise, using two different reflections to generate translations leads to the results $T_{1,2}: \Phi_{4,5,6} \rightarrow \Phi_{4,5,6}$. These are all in agreement with Table I.

We emphasize here that the above logic works because if two different reflection axes are related by a symmetry operation, then these two reflections should act on the (unique) monopole $\mathcal{M}$ in the same way. More precisely, $g\left(\mathcal{C R}_{1}\right) g^{-1}=\mathcal{C} \mathcal{R}_{1}$ when acting on a one-dimensional representation.

\section{Square}

As discussed before, it suffices to consider the $\pi$-flux phase, which has a charge conjugation symmetry. Now, consider $\mathcal{C} \mathcal{R}_{1} \equiv \mathcal{C} R_{x}, \mathcal{C} R_{2} \equiv \mathcal{C} T_{1} C_{4}^{2} R_{x}$, and $\mathcal{C} R_{3} \equiv$ $\mathcal{C} C_{4} T_{1} C_{4}^{2} R_{x}$-the last two are reflections across axes labeled in Fig. 4.

It is easy to check that $\left(\mathcal{C} R_{1}\right)^{2}=\left(C R_{3}\right)^{2}=-1$ and $\left(\mathcal{C} R_{2}\right)^{2}=+1$ on the fermions, which immediately leads to

$$
\begin{aligned}
& \mathcal{C} \mathcal{R}_{1}: \mathcal{M} \rightarrow \mathcal{M}, \\
& \mathcal{C R}_{2}: \mathcal{M} \rightarrow-\mathcal{M}, \\
& \mathcal{C R}_{3}: \mathcal{M} \rightarrow \mathcal{M} .
\end{aligned}
$$

It is now straightforward to read off other symmetry transformations on this monopole, $\mathcal{M} \sim \Phi_{4}+i \Phi_{5}$. For example, $C_{4} \sim \mathcal{C R}_{3} \cdot \mathcal{C R}_{1}: \mathcal{M} \rightarrow \mathcal{M}$ and $T_{1,2} \sim \mathcal{C R}_{1}$. $\mathcal{C R}_{2}: \mathcal{M} \rightarrow-\mathcal{M}$.

We also make a phase choice so that

$$
\mathcal{C}: \mathcal{M} \rightarrow \mathcal{M}^{\dagger}
$$

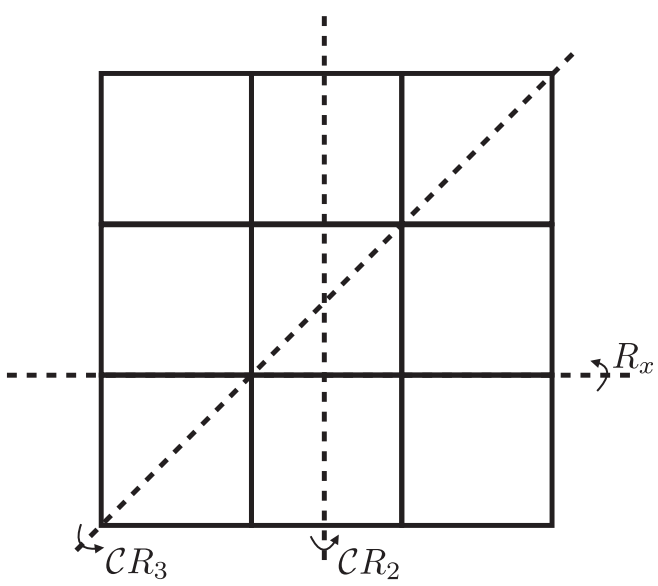

FIG. 4. Reflection axis to be considered in the main text.

The transformation of charge conjugation $\mathcal{C}$ depends on the phase choice of the monopole, but the relative phases between six monopoles are fixed and hence meaningful.

Then, we have essentially reproduced Table II for the $\pi$-flux state. The transformation for $\Phi_{1,2,3}$ can be fixed by further applying the emergent $S O(6)$ symmetry.

\section{A MORE GENERAL SCHEME: ATOMIC (WANNIER) CENTERS}

We have seen that on bipartite lattices (honeycomb and square), with the help of particle-hole symmetry (a hallmark of bipartite lattices), the monopole quantum numbers under lattice rotation and translation can be uniquely fixed. Here, we discuss a method applicable on all lattices including nonbipartite ones such as triangular and kagome types.

Notice that in this problem, lattice rotation plays a more fundamental role than lattice translation in two ways. First, if lattice rotation symmetry is absent, there will be no reason for the monopole to have a quantized lattice momentum, which can take a continuous value in the entire Brillouin zone. Now, if we impose certain rotation symmetry $C_{n}(n=2,3,4,6)$, the momentum will only take certain (discrete) rotationally invariant values, which will be robust as the system is adiabatically deformed. Second, once we know how the monopoles transform under rotations around different centers, we know automatically how it should transform under translation since translation operation can be generated by subsequent rotations around different centers.

Presumably, the lattice momentum and angular momentum of the monopole operator are also decided by the band topology of the spinon insulator, just like time-reversal symmetry-but how? Before answering this question systematically, let us consider a more familiar example of bosonic spinon (Schwinger boson) theory, where it is well known that on bipartite lattices the monopole carries $l= \pm 1$ angular momentum under site-centered rotations, 
leading to valence bond solid (VBS) order when the spinons are gapped $[29,30]$ - a fact that is important in the context of deconfined quantum phase transition between Néel and VBS states [31,32]. We now review this fact with a physically intuitive picture, which could then be generalized to more complicated cases with fermionic spinons. For the fermionic spinons, we illustrate the new method in this section with the bipartite examples. We obtain the same results as in Sec. IV, with considerably more involved calculations- the goal being to establish the method in a setting where the answers are independently known, so that we can apply it to the DSL on nonbipartite lattices (and further confirm the results on bipartite lattices).

\section{A. Warm-up: Bosonic spinons and the valence bond solid}

For concreteness, let us consider a honeycomb lattice with spin-1/2 per site. In the Schwinger boson formulation, we decompose the spin operator as

$$
\mathbf{S}_{i}=(-1)^{i} \frac{1}{2} b_{i, \alpha}^{\dagger} \sigma_{\alpha \beta} b_{i, \beta},
$$

where $i$ is the site label and $(-1)^{i}$ takes \pm 1 on the two sublattices, respectively. Note that $b_{\alpha}$ is a hard-core boson $\left[\left(b_{\alpha}^{\dagger}\right)^{2}=0\right]$ with spin $\alpha \in\{\uparrow, \downarrow\}$. The physical Hilbert space has $\sum_{\alpha} b_{\alpha}^{\dagger} b_{\alpha}=1$. Similar to the fermionic spinon theory, this constraint only needs to be satisfied, on average, in a spin-liquid phase. There is again a dynamical $U(1)$ gauge field $a_{\mu}$ coupled to the Schwinger bosons, with gauge charge on each site defined as $q_{i}=\sum_{\alpha} b_{\alpha}^{\dagger} b_{\alpha}-1$.

The $(-1)^{i}$ factor in the parton decomposition is chosen so that when the Schwinger bosons condense in a uniform manner, the spins order as a collinear antiferromagnet (Néel state). Because of this $(-1)^{i}$ factor, under the $C_{6}$ rotation (which exchanges the two sublattices), the Schwinger bosons should transform (in addition to the coordinate change) as

$$
b_{\alpha} \rightarrow i \sigma_{\alpha \beta}^{2} b_{\beta}^{\dagger} \text {. }
$$

A similar transform also happens on the square lattice under translation.

We would like to construct a state in which the Schwinger bosons are gapped; i.e., they form a bosonic Mott insulator. The simplest such state respecting all the symmetries - especially spin rotation and $C_{6}$ rotation-is shown pictorially in Fig. 5. In this state, every site in sublattice $A$ is empty, and every site in sublattice $B$ has both bosonic orbits occupied. The wave function is simply a product state,

$$
\prod_{i \in A}|0\rangle_{i} \prod_{j \in B} b_{j, \uparrow}^{\dagger} b_{j, \downarrow}^{\dagger}|0\rangle_{j} .
$$

Now, what happens when a monopole (a flux quantum) moves in this simple charge background? What the flux

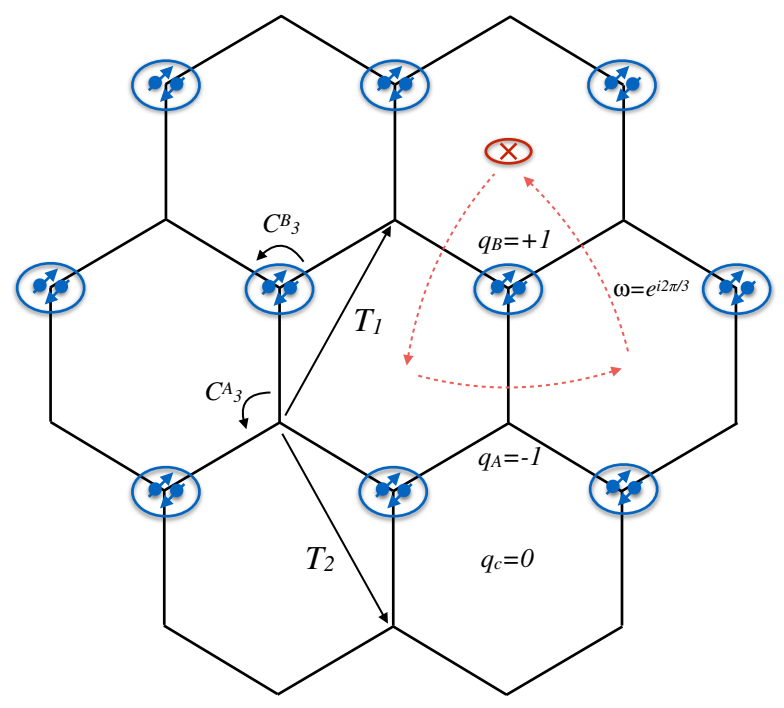

FIG. 5. The simplest spinon (boson) Mott insulator, with every site in sublattice $A$ empty and every site in sublattice $B$ completely filled (recall that the bosons $b_{\alpha}$ are hard core). This case respects $C_{6}$ rotation since it acts as $b_{\alpha} \rightarrow i \sigma_{\alpha \beta}^{y} b_{\beta}^{\dagger}$. As a monopole (flux) moves in this background, it sees a fixed gauge charge pattern with $q_{A}=-1$ and $q_{B}=+1$. The amount of gauge charge sitting on each rotation center dictates the nontrivial Berry phase accumulated by the monopole as it moves around the center according to $\theta\left(C_{n}^{r}\right)=e^{i q, 2 \pi / n}$. The translation quantum numbers of the monopole can be easily obtained once the rotation quantum number is known.

sees is a fixed gauge charge pattern, with $q_{A}=-1$ on each $A$ site and $q_{B}=+1$ on each $B$ site. Therefore, as the flux moves around each site, a nontrivial Berry phase is picked up. The amount of gauge charge $q_{r}$ sitting on each rotation center $r$ dictates the Berry phase under the $C_{n}$ rotation to be

$$
\theta\left(C_{n}^{r}\right)=e^{i q_{r} 2 \pi / n} .
$$

Effectively, the monopole gains an angular momentum $l_{A}=-1$ under $A$-site-centered $C_{3}^{A}$ rotation (corresponding to a Berry phase $\omega^{-1}=e^{-i 2 \pi / 3}$ under a $C_{3}^{A}$ rotation), $l_{B}=$ +1 under $B$-site-centered $C_{3}^{B}$ rotation (Berry phase $\omega$ ), and $l_{c}=0$ under plaquette-centered $C_{6}^{c}$ rotation since there is no charge placed at the center of the hexagon plaquettes. One may ask how robust these Berry phases are-for example, will the results change if the flux is inserted far away from the rotation center? The answer is no since by $C_{n}$ symmetry the total charge enclosed by a closed rotation trajectory will always be $q_{r}(\bmod n)$. Therefore, the monopole Berry phase will always be given by Eq. (38) under a $C_{n}$ rotation. This case is essentially the spirit of dimensional reduction introduced in Ref. [58].

Translation symmetry quantum numbers are now easily obtained: $T_{1}=C_{A}^{-1} C_{B}=\omega^{2}$ and $T_{2}=C_{B} C_{A}^{-1}=\omega^{2}$. This case makes the monopole operator the Kakule VBS order parameter, as expected. 
The above argument can be extended straightforwardly to the square lattice, from which the standard results follow; namely, the monopole carries \pm 1 angular momentum under site-centered $C_{4}$ rotations and is therefore identified with a columnar VBS order parameter $[29,30]$.

\section{B. Wannier centers on the honeycomb lattice: A case study}

We now return to fermionic spinons in a quantum spin Hall insulator band structure. The reason to use quantum spin Hall mass follows from discussions in Sec. III, as it preserves the spatial symmetries of interest. Let us first consider the simpler honeycomb lattice. The band structure is given by the Haldane model [59] for each spin component with opposite Chern numbers. The hopping amplitudes include a real nearest-neighbor hopping and an imaginary second-neighbor hopping that preserve all the lattice symmetries and time-reversal symmetry $f \rightarrow i \sigma^{2} f$. One may ask whether we can deform this insulator to an "atomic limit" as we did above in Sec. VA and then trivially read off the monopole's lattice quantum numbers. Obviously, this is impossible if time-reversal and spin rotation [now only $S O(2)$ ] symmetries are preserved since the spinons form a strong topological insulator, and therefore (almost by definition) an atomic limit does not exist. However, since the question of monopole lattice quantum number has nothing to do with spin rotation and timereversal symmetry, we may as well explicitly break all the symmetries except the lattice translation, rotation, and the $U(1)$ charge conservation. In the simplest setting, the resulting insulator can be deformed into an "atomic limit," which can be pictured as particles completely localized in real space. One nontrivial aspect, compared to the bosonic spinon case in Sec. VA, is that the effective centers of the localized orbits do not have to sit on the original lattice sites (where the microscopic fermions are defined). For insulators described by free-fermion band theory, these are simply the Wannier centers. One can always deform all the Wannier centers, without further breaking any symmetry, to one of the rotation centers, such as lattice sites or plaquette centers (see Fig. 6, for example). Once such a configuration is reached, we can simply follow the procedure in Sec. VA to obtain the monopole Berry phase under a $C_{n}$ rotation centered at $r$ according to Eq. (38). Lattice momentum of the monopole can then be obtained by composing rotations at different centers.

Let us illustrate this with the QSH insulator on the honeycomb lattice - for concreteness, we assume that the nearest-neighbor hopping $t>0$. To determine the exact nature of the Wannier limit, we employ the techniques developed in Refs. [36-38]. The basic idea is to look at high symmetry points in momentum space, namely, momentum points that are invariant under certain lattice rotations. The fermion Bloch states at these high symmetry points will now form certain representations of the rotation

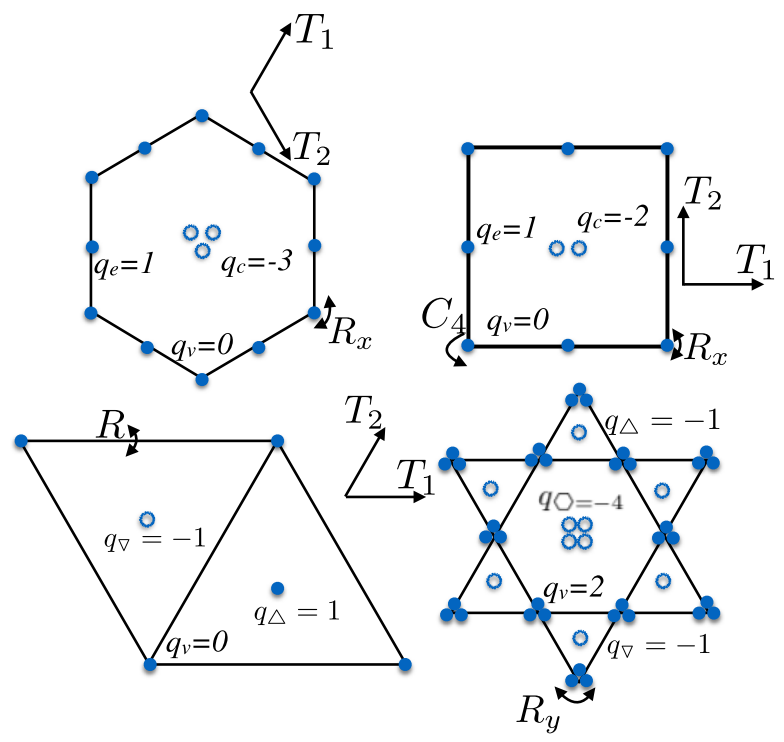

FIG. 6. The Wannier limit of a quantum spin Hall insulator on four types of lattices when all the symmetries are broken except for charge $U(1)$ conservation and lattice translation or rotation. The solid dot indicates a Wannier center, and empty dot indicates a "minus" charge pertinent to the fragile nature of the band topology. The pattern of $U(1)$ gauge charge is shown in the figure denoted as $q$, with self-explanatory subscripts associated with locations of charges (e.g., $e, c, v$ mean charges located at center of edges, plaquettes, on vertices, respectively), with the background -1 charge on-site subtracted.

symmetries. Different atomic insulators will also have Bloch states at high symmetry momentum points forming certain symmetry representations. Our task is to determine what combination of atomic (Wannier) limit would give rise to the representations of the spinon band of our interest.

Consider inversion symmetries $I_{2}^{c}$ with respect to a hexagon center. There are four inversion-invariant momentum points: $\Gamma\left(T_{1}=T_{2}=1\right), M\left(T_{1}=T_{2}=-1\right)$, and two other points $M^{\prime}, M^{\prime \prime}$ related to $M$ by $C_{6}$ rotations. Since there are in total two bands occupied (one per spin), the Bloch states form a two-dimensional reducible representation of $I_{2}^{c}$ at each of these momentum points. The spectrum of inversion eigenvalues $\left\{\lambda_{I^{c}}\right\}$ at each momentum is

$$
\begin{aligned}
& \lambda_{I^{c}}^{\Gamma}:\{+1,+1\}, \\
& \lambda_{I^{c}}^{M}:\{-1,-1\} .
\end{aligned}
$$

Now, what kind of atomic insulator can form such representations at $\Gamma$ and $M$ ? Let us consider several candidates. First, consider the simplest insulator, with exactly one Wannier center sitting on each lattice site. It is easy to show that this insulator has $\lambda_{I^{c}}:\{+1,-1\}$ at both $\Gamma$ and $M$. Next, consider an insulator with one Wannier center sitting at the center of each edge. This insulator has $\lambda_{I^{c}}^{\Gamma}: \lambda_{0} \times\{1,1,1\}$ and $\lambda_{I^{c}}^{M}: \lambda_{0} \times\{1,-1,-1\}$, where $\lambda_{0}$ is the intrinsic inversion eigenvalue of the Wannier orbit, which 
can be either \pm 1 (in more conventional language, these correspond to $s$ orbitals and $p$ orbitals on the bonds). There is yet another insulator with one Wannier center sitting at the center of each hexagon plaquette. This insulator has $\lambda_{I^{c}}^{\Gamma}=\lambda_{I^{c}}^{M}=\lambda_{0}$ for some intrinsic $\lambda_{0}= \pm 1$. We have exhausted all distinct types of atomic (Wannier) insulators, but it appears that none of these fits the representation of our occupied band Eq. (39). The key concept we need here is that of fragile topology [37]: The occupied band, even though it is "topologically trivial," can be deformed into an atomic (Wannier) limit only when combined with another atomic insulator. Formally, this means that we are allowed to consider "subtracting" representations at high symmetry momentum points that correspond to the "auxiliary" atomic insulators. The whole procedure then becomes solving a set of integer-valued linear equations, with the unknowns being the number of each atomic insulator, which can be either positive or negative (more details can be found in Refs. [36,37]). In our case, it turns out that if we combine the occupied band with an atomic insulator with three Wannier centers sitting on each hexagon center, with intrinsic inversion eigenvalues $\lambda_{0}=\{1,1,-1\}$, the total system will form inversion representations at $\Gamma$ and $M$ that resemble an atomic insulator with one Wannier center on each site and at the center of each edge (with $\lambda_{0}=1$ ). Formally, we can write this relation as (upper left of Fig. 6)

$$
\begin{aligned}
& \text { honeycomb QSH occupied band } \\
& \begin{array}{l}
=\text { site }+ \text { edge }^{\lambda_{0}=1} \\
\quad-2 \times \text { hexagon }^{\lambda_{0}=1}-\text { hexagon }^{\lambda_{0}=-1},
\end{array}
\end{aligned}
$$

where the minus signs indicate the fragile nature of the topology. From the monopole point of view, the bands that are formally subtracted in the above relation can simply be viewed as negative gauge charges sitting on their Wannier centers. So the above relation produces a gauge charge pattern shown in Fig. 6 (remember that there is always a -1 background gauge charge sitting on each site).

One should also check that the above Wannier pattern also reproduces representations at high symmetry points of other point group symmetries such as site-centered $C_{3}$. Since the $C_{3}$-invariant momentum points include the Dirac points $\left[\mathbf{K}=[(2 \pi / 3),(2 \pi / 3)], \mathbf{K}^{\prime}=-\mathbf{K}\right]$, it is important for us to include the Dirac mass term that corresponds to the QSH mass. It is a relatively straightforward exercise to check that the above Wannier pattern indeed reproduces the representations from the occupied band of the QSH insulator.

The gauge charge pattern thus produced gives rise to some nontrivial quantum numbers for the monopole. Using Eq. (38), we conclude that the monopole transforms under $C_{6}$ as $\mathcal{M} \rightarrow-\mathcal{M}$ and transforms trivially under lattice translations. This result is exactly what we found in Sec. IV and what we numerically found in Ref. [35].
We comment that we can alternatively focus on the unoccupied bands of the QSH insulator (holes) instead of the occupied bands (particles) and ask about Wannier centers of the holes. Since the occupied and unoccupied bands together produce two trivial Wannier orbits on each lattice site, it can be shown straightforwardly that we would obtain the identical gauge charge pattern by considering holes instead of particles.

\section{Wannier centers on the square lattice}

Now, consider the $\pi$-flux state on the square lattice. We deform the band structure to the QSH regime. One could obtain how monopoles transform under various rotations by deforming the spinon bands to a Wannier insulator as we did above on the honeycomb lattice. One finds that on the square lattice (upper right of Fig. 6),

$$
\begin{aligned}
& \text { square QSH occupied band } \\
& =\text { site }+ \text { edge center }-2 \times \text { plaquette },
\end{aligned}
$$

where the rhs means Wannier insulators with particles lying on site, edge center, or plaquette centers. Accounting for the background negative one gauge charge per site, this configuration means the gauge charge on-site vanishes, leading to spin-triplet monopoles staying invariant, under site-centered $C_{4}$ in Table II, while for $C_{4}$ around the plaquette center, the monopoles get -1 since they see two gauge charges sitting at the rotation center. This result implies that, for example, under $T_{1}, T_{2}$ the spin-triplet monopoles should change sign, in agreement with Table II.

We note that similar results were obtained in Ref. [40] by considering a VBS insulator formed by the spinons, in which the effective Wannier centers are simply sitting on the lattice sites and the computation is simpler.

\section{MONOPOLE QUANTUM NUMBERS II: NONBIPARTITE LATTICES}

We are finally ready to calculate monopole lattice quantum numbers on the nonbipartite lattices, i.e., triangular and kagome lattices.

\section{A. Triangular lattice: Wannier centers from projective symmetry group}

\section{Mean field and PSG}

On triangular lattice, we focus on a particular mean-field ansatz with the Hamiltonian

$$
\mathcal{H}=J \sum_{\langle i j\rangle} t_{i j} \sum_{\alpha} f_{i \alpha}^{\dagger} f_{j \alpha}+\text { H.c. },
$$

where $t_{i j}= \pm 1$ and there is a "staggered $\pi$-flux" configuration of $t_{i j}$ on the triangular lattice, with a $\pi$ flux on each upward triangle and zero flux on each downward triangle. 
More details can be found in Appendix A. Under an appropriate basis, the low-energy Hamiltonian takes the standard Dirac form, with two spins (denoted by Pauli matrices $\sigma$ ) and two valleys (denoted by Pauli matrices $\tau$ ). The projective symmetry representation of the Dirac fermions (translation $T_{1 / 2}$, reflection $R_{x}$, sixfold rotation $C_{6}$, and time reversal) are calculated in the standard method, and the results are listed below:

$$
\begin{aligned}
& \psi \stackrel{T_{1}}{\rightarrow} i \tau^{3} \psi, \\
& \psi \stackrel{T_{2}}{\rightarrow}-i \tau^{2} \psi, \\
& \psi \stackrel{\mathcal{T}}{\rightarrow} i \sigma^{2} \mu^{2} \tau^{2} \psi(-k), \\
& \psi \stackrel{C_{6}}{\rightarrow} i \sigma^{2} W_{C_{6}} \psi^{\dagger}, \\
& \psi \stackrel{R}{\rightarrow} i \sigma^{2} W_{R} \psi^{\dagger},
\end{aligned}
$$

where we have suppressed the coordinate transforms and

$$
\begin{aligned}
W_{C_{6}} & =e^{-i \gamma^{0}(\pi / 6)} W_{c} \sigma^{2} e^{i(\pi / 3) \tau^{c}} \quad \tau^{C}=\frac{1}{\sqrt{3}}\left(\tau^{1}+\tau^{2}+\tau^{3}\right), \\
W_{R} & =\frac{\left(\gamma^{1}-\sqrt{3} \gamma^{2}\right)}{2} W_{c} \frac{\tau^{3}-\tau^{1}}{\sqrt{2}}, \\
W_{c} & =\frac{1}{\sqrt{3}}\left(-i I_{4 \times 4}-\mu^{3}+\mu^{1}\right),
\end{aligned}
$$

where $\mu^{i}$ are Pauli matrices acting on the Dirac spinor index (i.e., superposition of $\gamma$ matrices). Again, more details can be found in Appendix A. We remark that the $C_{6}$ symmetry operation involves an extra charge conjugation [which is part of the $S U(2)$ gauge symmetry on spinons] due to the staggered flux pattern in the mean-field ansatz.

The above transformations on Dirac fermions fix the monopole transformations up to overall $U(1)_{\text {top }}$ phase factors. The results are listed in Table III. Let us provide some more explanations here:

(i) Translations $T_{1 / 2}: \psi \stackrel{T_{1}}{\rightarrow}-i \tau^{2} \psi, \psi \stackrel{T_{2}}{\rightarrow} i \tau^{3} \psi$, which give a relative minus sign to $\Phi_{1 / 3}, \Phi_{1 / 2}$, respectively. The overall phase factor is undetermined, but it is

TABLE III. Monopole transformation laws on the triangular lattice. Note that $C_{6}$ is sixfold rotation around the site, and other symmetries are marked in Fig. 6. There are nontrivial Berry phases for translations.

\begin{tabular}{lccccc}
\hline \hline & $T_{1}$ & $T_{2}$ & $R$ & $C_{6}$ & $\mathcal{T}$ \\
\hline$\Phi_{1}^{\dagger}$ & $e^{i(-\pi / 3)} \Phi_{1}^{\dagger}$ & $e^{i(\pi / 3)} \Phi_{1}^{\dagger}$ & $-\Phi_{3}^{\dagger}$ & $\Phi_{2}$ & $\Phi_{1}$ \\
$\Phi_{2}^{\dagger}$ & $e^{i(2 \pi / 3)} \Phi_{2}^{\dagger}$ & $e^{i(\pi / 3)} \Phi_{2}^{\dagger}$ & $\Phi_{2}^{\dagger}$ & $-\Phi_{3}$ & $\Phi_{2}$ \\
$\Phi_{3}^{\dagger}$ & $e^{i(-\pi / 3)} \Phi_{3}^{\dagger}$ & $e^{i(-2 \pi / 3)} \Phi_{3}^{\dagger}$ & $-\Phi_{1}^{\dagger}$ & $-\Phi_{1}$ & $\Phi_{3}$ \\
$\Phi_{4 / 5 / 6}^{\dagger}$ & $e^{i(2 \pi / 3)} \Phi_{4 / 5 / 6}^{\dagger}$ & $e^{i(-2 \pi / 3)} \Phi_{4 / 5 / 6}^{\dagger}$ & $\Phi_{4 / 5 / 6}^{\dagger}$ & $-\Phi_{4 / 5 / 6}$ & $-\Phi_{4 / 5 / 6}$ \\
\hline \hline
\end{tabular}

constrained by the invariance of $\Phi_{4 / 5 / 6}$ under $C_{3}=$ $C_{6}^{2}$ to take a value in $\left\{1, \omega \equiv e^{i(2 \pi / 3)}, \omega^{-1}\right\}$.

(ii) Time reversal $\mathcal{T}: \psi \stackrel{\mathcal{T}}{\rightarrow} i \sigma^{2} \mu^{2} \tau^{2} \psi=\mathcal{T}_{0} \psi$. From Sec. II, we know that $\Phi \rightarrow O_{T} \Phi^{\dagger}$, which fixes the overall phase factor.

(iii) Sixfold rotation around site $C_{6}$ :

$$
\psi \stackrel{C_{6}}{\rightarrow} e^{-i \gamma_{0}(\pi / 6)}\left\{i \sigma^{2} W_{c} \tau^{2}\right\} \exp \left[i \frac{\pi}{3} \tau^{C}\right] \psi^{*},
$$

where the part inside \{\} is identical to $\mathcal{C}_{0}$ defined in Eq. (13). We disregard the first Lorentz rotation since the monopole is a scalar anyway. The last part involves certain $S O(3)$ rotations in $\Phi_{1 / 2 / 3}$ induced by $\tau^{2}\left[\frac{1}{2}\left(I_{4 \times 4}-i \tau^{3}-i \tau^{2}-i \tau^{1}\right)\right]$. From Sec. II, we know that $\mathcal{C}_{0}: \Phi \rightarrow \pm O_{T} \Phi^{\dagger}$, where the overall phase depends on the convention. Fixing the overall phase, we get the last column in Table III.

(iv) Reflection $R_{x}$ :

$$
\psi \stackrel{R}{\rightarrow} \frac{\left(\gamma_{1}-\sqrt{3} \gamma_{2}\right)}{2}\left\{i \sigma^{2} W_{c} \tau^{2}\right\} \tau^{2} \frac{\tau^{3}-\tau^{1}}{\sqrt{2}} \psi^{*} .
$$

This formula is really a $\mathcal{C R}$ symmetry. Since quantum spin Hall mass $\bar{\psi} \sigma \psi$ stays invariant under $R$, we could invoke the reflection-protected topological phase argument in Sec. III. It is easy to check that $R^{2}=(-1)^{F}$, which means that $R: \Phi_{4 / 5 / 6} \rightarrow$ $\Phi_{4 / 5 / 6}$ since this is not a nontrivial $R$ protected topological phase. To fix how $\Phi_{1 / 2 / 3}$ transform requires a bit of caution: First, they differ from $\Phi_{4 / 5 / 6}$ transformations by a minus sign from $\mathcal{C}_{0}$ (the part encoded in big brackets in the transformation); on top of that, they are rotated by $\tau^{2}\left(\tau^{3}-\tau^{1} / \sqrt{2}\right)$. Combining these steps, one gets the full reflection transformation.

So at this point, the only unfixed phase factors are those associated with $T_{1,2}$. We now calculate these phases using the Wannier center technique developed in Sec. V.

\section{Monopole angular momenta from Wannier centers}

Below, we calculate the Wannier centers in the triangular lattice setting to deduce the monopole angular momenta for rotations around different centers. We use the approach described in Sec. V and consider three kinds of threefold rotations around site $C_{3}^{\circ}$, upward-triangle center $C_{3}^{\triangle}$, and downward-triangle center $C_{3}^{\nabla}$, respectively. To obtain the Wannier limit for the DSL spinon band structure with quantum spin Hall mass as discussed in Sec. V, we first calculate representations for the rotation groups at high symmetry momenta.

First, one takes a four-site unit cell with a $C_{3}$-invariant Brillouin zone illustrated in Fig. 7. Under the three types of 
triangles is always of the form (under an appropriate phase choice of the superposition coefficients)

$$
\left(\begin{array}{lll}
0 & 1 & 0 \\
0 & 0 & 1 \\
1 & 0 & 0
\end{array}\right),
$$

whose eigenvalue is $\left\{1, \omega, \omega^{2}\right\},\{\omega \equiv \exp [i(2 \pi / 3)]\}$; the nine-dimensional subspace spanned by Wannier functions of $\triangle_{A / B / D}$ at high symmetry points therefore constitute a representation with eigenvalues $3 \times\left\{1, \omega, \omega^{2}\right\}$, where 3 means a direct sum of the set of eigenvalues. On the other hand, $\triangle_{C}$ goes to its equivalent under $C_{3}^{\circ}$. Consider the Wannier function localized at $\triangle_{C}$ :

$$
\psi\left(\triangle_{C}\right)=\frac{1}{\sqrt{3}}(-|1\rangle+|2\rangle-|3\rangle),
$$

where the site labels are marked in Fig. 7, under $C_{3}^{\circ}$,

$$
\psi\left(\triangle_{C}\right) \rightarrow \psi\left(\triangle_{C^{\prime}}\right)=-\frac{1}{\sqrt{3}}\left(-\left|1^{\prime}\right\rangle+\left|2^{\prime}\right\rangle-\left|3^{\prime}\right\rangle\right) .
$$

Since $C, C^{\prime}$ differ by a lattice vector $\mathbf{r}_{2}$, the eigenvalue reads $\exp \left[i k(\vec{M}) \cdot \vec{r}_{2}\right]$. Similarly, for the other two $\triangle_{C}$ wave functions with angular momentum $\eta_{1}$,

$\psi\left(\triangle_{C}\right)=\frac{1}{\sqrt{3}}\left(-|1\rangle+\omega^{\eta_{1}}|2\rangle-\omega^{2 \eta_{1}}|3\rangle\right) \quad\left(\eta_{1}=1,2\right)$,

the eigenvalues are $-\omega^{\eta_{1}} \exp \left[i \mathbf{k}(\mathbf{M}) \mathbf{r}_{2}\right]$.

One combines the above diagonalized representation for $\triangle_{A / B / D}$ with that of the $\triangle_{C}$ wave function of certain angular momentum $\eta_{1}$, leading to the second column, second row of Table IV. We proceed in a similar fashion to produce the rest of the table.

Comparing the representation of spinon bands with those of the three Wannier insulators, we identify a unique decomposition of the spinon bands into Wannier centers (lower left of Fig. 6):

$$
\text { triangular QSH occupied band }=\text { site }+\triangle-\nabla,
$$

where again the minus sign denotes a formal difference in light of the fragile topology. Taking into account the background gauge charge -1 per site, the spin-triplet monopoles see no gauge charge rotating around site and \pm 1 gauge charge rotating around an upward- or downwardtriangle center, respectively. Since $T_{2}=\left(C_{3}^{\nabla}\right)^{-1} C_{3}^{\Delta}$ with two rotation centers located in one unit cell shown in Fig. 7, monopoles $\mathcal{S}_{i}$ transform with a phase $-(-2 \pi / 3)+$ $(2 \pi / 3)=(4 \pi / 3)$, and similarly, they get a phase of $\omega$ under $T_{1}$. To sum up, we get the transformation of monopoles as tabulated in Table III.

Sometimes it is not sufficient to only match the eigenvalues because of the complicated PSG structure. A full calculation would be to compare the full representations of lattice PSG (rotation and translation) at high symmetry points in the Brillouin zone, which are generically nonAbelian. We do not perform such a full calculation here since the much simpler calculation is already sufficiently constraining to fix the Wannier centers; i.e., only the Wannier insulator composition in Eq. (52) has the same rotation eigenvalues at high symmetry points as those of the triangular Dirac spin liquid with QSH mass; hence, their full representations must also match.

\section{B. Kagome lattice}

On the kagome lattice, similar to the triangular case, Ref. [9] calculated the kagome DSL with a staggered flux mean-field ansatz, with three gamma matrices as $\gamma_{\nu}=\left(\mu^{3}, \mu^{2},-\mu^{1}\right)$, and we have, for the PSG of Dirac fermions,

$$
\begin{aligned}
& T_{1}: \psi \rightarrow\left(i \tau^{2}\right) \psi \quad T_{2}: \psi \rightarrow\left(i \tau^{3}\right) \psi \\
& R_{y}: \psi \rightarrow\left(i \mu^{1}\right) e^{(i \pi / 2) \tau_{r y} \psi} \\
& C_{6}: \psi \rightarrow e^{(i \pi / 3) \mu^{3}} e^{(2 \pi i / 3) \tau_{R}} \psi \\
& \mathcal{T}: \psi \rightarrow\left(i \sigma^{2}\right)\left(i \mu^{2}\right)\left(-i \tau^{2}\right) \psi,
\end{aligned}
$$

where

$\tau_{r y}=\frac{-1}{\sqrt{2}}\left(\tau^{1}+\tau^{3}\right), \quad \tau_{R}=\frac{1}{\sqrt{3}}\left(\tau^{1}+\tau^{2}-\tau^{3}\right)$.

The PSG again fixes the monopole transformations, up to overall phase factors to be determined, as listed in Table V. The overall phase for time reversal is fixed through the argument in Sec. II. The overall phase for $R_{y}$ is convention dependent, and we fix it as in Table V. The fact that $\Phi_{4,5,6}$ are invariant (up to a phase) under $C_{6}$ requires their momenta to be zero, which in turn fixes the overall phases associated with $T_{1,2}$ as in Table V. The only undetermined phase is that in $C_{6}$, which we fix below.

TABLE V. The transformation of monopoles on the kagome lattice. Here, $R_{y}, C_{6}$ denote the reflection perpendicular to the vertical direction and sixfold rotation around centers of the hexagon in Fig. 6.

\begin{tabular}{lccccc}
\hline \hline & $T_{1}$ & $T_{2}$ & $R_{y}$ & $C_{6}$ & $\mathcal{T}$ \\
\hline$\Phi_{1}^{\dagger}$ & $-\Phi_{1}^{\dagger}$ & $-\Phi_{1}^{\dagger}$ & $-\Phi_{3}$ & $e^{i(2 \pi / 3)} \Phi_{2}^{\dagger}$ & $\Phi_{1}$ \\
$\Phi_{2}^{\dagger}$ & $\Phi_{2}^{\dagger}$ & $-\Phi_{2}^{\dagger}$ & $\Phi_{2}$ & $-e^{i(2 \pi / 3)} \Phi_{3}^{\dagger}$ & $\Phi_{2}$ \\
$\Phi_{3}^{\dagger}$ & $-\Phi_{3}^{\dagger}$ & $\Phi_{3}^{\dagger}$ & $-\Phi_{1}$ & $-e^{i(2 \pi / 3)} \Phi_{1}^{\dagger}$ & $\Phi_{3}$ \\
$\Phi_{4 / 5 / 6}^{\dagger}$ & $\Phi_{4 / 5 / 6}^{\dagger}$ & $\Phi_{4 / 5 / 6}^{\dagger}$ & $-\Phi_{4 / 5 / 6}$ & $e^{i(2 \pi / 3)} \Phi_{4 / 5 / 6}^{\dagger}$ & $-\Phi_{4 / 5 / 6}$ \\
\hline \hline
\end{tabular}


TABLE VI. The space group representation of rotations on the kagome lattice at high symmetry points $\mathbf{k}=[(2 \pi / 3),(\pi / 3)]$, $\mathbf{Q}=(0, \pi)$. Superscripts $\triangle, \nabla, \square$ denote rotations around upward- and downward-triangle, and hexagon centers, respectively. We have $\Omega=e^{i(\pi / 6)}, \omega=e^{i 2 \pi / 3}$, and [Sextet] $=i\left[e^{ \pm i(\pi / 6)}, \pm i, e^{ \pm i(5 \pi / 6)}\right]$, and integers $\eta_{1,2,3}$ are orbital angular momenta of Wannier functions located at centers of upward or downward triangles and hexagons, respectively. Here, $p_{1}=e^{i \mathbf{k}(\mathbf{Q}) \cdot r_{1}}$ is the phase factor under $T_{1}$ translation at the high symmetry momenta of interest, and $\Gamma^{\triangle}, \Gamma^{\nabla}, \Gamma^{\circ}, \Gamma^{\circ}$ denote representations of Wannier functions centered at the upward or downward triangles, sites, and hexagons, respectively. For $\Gamma_{k}^{\mathrm{PSG}}$, there are two sets of fourfold degenerate states, which can be simultaneously block diagonalized into two $2 \times 2$ matrices for all three $C_{3}$ rotations, and we list the pairwise eigenvalues in brackets. The multiplicity of reps. $(>1)$ is denoted as a positive integer $\times$ the particular rep. The Wannier limit of the spinon band in the last two rows is derived using the spinon band with a quantum spin Hall mass added.

\begin{tabular}{|c|c|c|c|c|c|c|}
\hline Symmetry & $\Gamma_{k / Q}^{\triangle}$ & $\Gamma_{k / Q}^{\nabla}$ & $\Gamma_{k / Q}^{\circ}$ & $\Gamma_{k / Q}^{\circ}$ & $\begin{array}{c}\Gamma_{k}^{\mathrm{PSG}} \text { (fourfold particle } \\
\oplus \text { twofold particle } \\
\oplus \text { fourfold hole } \oplus \text { twofold hole) }\end{array}$ & $\begin{array}{c}\Gamma_{Q}^{\mathrm{PSG}}[\text { fourfold particle } \\
\oplus \text { fourfold }(\text { Dirac fermion }) \\
\oplus \text { fourfold hole }]\end{array}$ \\
\hline$C_{3}^{\triangle}$ & $\begin{array}{c}\omega^{\eta_{1}} \\
\oplus\left[1, \omega, \omega^{2}\right]\end{array}$ & $\begin{array}{c}\omega^{\eta_{2}} p_{1} \\
\oplus\left[1, \omega, \omega^{2}\right]\end{array}$ & $\begin{array}{c}\omega^{\eta_{3}} p_{1}^{*} \\
\oplus\left[1, \omega, \omega^{2}\right]\end{array}$ & $4 \times\left[1, \omega, \omega^{2}\right]$ & $\begin{array}{c}{\left[\left(\omega, \omega^{2}\right),(1, \omega)\right] \oplus\left[1, \omega^{2}\right]} \\
\oplus\left[\left(\omega, \omega^{2}\right),(1, \omega)\right] \oplus\left[1, \omega^{2}\right]\end{array}$ & $\begin{array}{c}{\left[\omega, \omega, \omega^{2}, \omega^{2}\right] \oplus\left[1,1, \omega, \omega^{2}\right]} \\
\oplus\left[1,1, \omega, \omega^{2}\right]\end{array}$ \\
\hline$C_{3}^{\nabla}$ & $\begin{array}{c}\omega^{\eta_{1}} p_{1}^{*} \\
\oplus\left[1, \omega, \omega^{2}\right]\end{array}$ & $\begin{array}{c}\omega^{\eta_{2}} \\
\oplus\left[1, \omega, \omega^{2}\right]\end{array}$ & $\begin{array}{c}\omega^{\eta_{3}} p_{1} \\
\oplus\left[1, \omega, \omega^{2}\right]\end{array}$ & $4 \times\left[1, \omega, \omega^{2}\right]$ & $\begin{array}{c}{\left[\left(1, \omega^{2}\right),\left(\omega^{2}, \omega\right)\right] \oplus[1, \omega]} \\
\oplus\left[\left(1, \omega^{2}\right),\left(\omega^{2}, \omega\right)\right] \oplus[1, \omega]\end{array}$ & $\begin{array}{c}{\left[\omega, \omega, \omega^{2}, \omega^{2}\right] \oplus\left[1,1, \omega, \omega^{2}\right]} \\
\oplus\left[1,1, \omega, \omega^{2}\right]\end{array}$ \\
\hline$C_{3}^{\circ}$ & $\begin{array}{c}\omega^{\eta_{1}} p_{1} \\
\oplus\left[1, \omega, \omega^{2}\right]\end{array}$ & $\begin{array}{c}\omega^{\eta_{2}} p_{1}^{*} \\
\oplus\left[1, \omega, \omega^{2}\right]\end{array}$ & $\begin{array}{c}\omega^{\eta_{3}} \\
\oplus\left[1, \omega, \omega^{2}\right]\end{array}$ & $4 \times\left[1, \omega, \omega^{2}\right]$ & $\begin{array}{c}{\left[(\omega, 1),\left(1, \omega^{2}\right)\right] \oplus\left[\omega, \omega^{2}\right]} \\
\oplus\left[(\omega, 1),\left(1, \omega^{2}\right)\right] \oplus\left[\omega, \omega^{2}\right]\end{array}$ & $\begin{array}{c}{\left[\omega, \omega, \omega^{2}, \omega^{2}\right] \oplus\left[1,1, \omega, \omega^{2}\right]} \\
\oplus\left[1,1, \omega, \omega^{2}\right]\end{array}$ \\
\hline$C_{6}$ & $\begin{array}{l}{\left[ \pm \Omega^{2 \eta_{1}+1}\right]} \\
\oplus[\text { Sextet }]\end{array}$ & $\begin{array}{l}{\left[ \pm \Omega^{2 \eta_{2}+1}\right]} \\
\oplus[\text { Sextet }]\end{array}$ & $\begin{array}{c}\Omega^{2 \eta_{3}+1} \oplus(-1)^{\eta_{3}} \\
{\left[e^{i(\pi / 6)}, e^{i(5 \pi / 6)},-i\right]}\end{array}$ & $2 \times[$ Sextet $]$ & $\Gamma_{Q}^{\mathrm{PSG}}:\left[\Omega, \Omega^{5}, \Omega^{-5}, \Omega^{*}\right] \oplus[ \pm i$ & $\left., \Omega, \Omega^{*}\right] \oplus\left[ \pm i, \Omega^{5}, \Omega^{-5}\right]$ \\
\hline \multicolumn{7}{|c|}{$\begin{array}{l}\Gamma^{\text {particle }}=3 \Gamma_{L=0}^{\circ}-\Gamma_{L=0}^{\triangle}-\Gamma_{L=0}^{\nabla}-\Gamma_{L=0}^{\circ}-\Gamma_{L=3}^{\bigcirc}-\Gamma_{L=4}^{\bigcirc}-\Gamma_{L=5}^{\bigcirc} \\
\Gamma^{\text {hole }}=-\Gamma_{L=0}^{\circ}+\Gamma_{L=0}^{\triangle}+\Gamma_{L=0}^{\nabla}+\Gamma_{L=0}^{\circ}+\Gamma_{L=3}^{\circ}+\Gamma_{L=4}^{\bigcirc}+\Gamma_{L=5}^{\bigcirc}\end{array}$} \\
\hline
\end{tabular}

The calculation is essentially the same as in the triangular case, so we will be brief here.

One calculates symmetry representations of threefold rotation around upward- or downward-triangle centers, and sixfold rotation around hexagon centers, as listed in Table VI. The spinon band is represented as (lower right of Fig. 6)

kagome QSH occupied band $=3$ site $-\triangle-\nabla-4 \square$

where $\triangle, \nabla, \square$ denote Wannier insulators localized on upward or downward triangles, and hexagons. Note that the numeral factor only denotes the numbers of occupied particles localized at the Wannier center; they may have different Wannier wave functions. This means the $C_{6}$ rotation sees a $(-4 \pi / 3)$ Berry phase, and translation begets no Berry phase since translation is composed of $C_{3}^{\triangle}\left(C_{3}^{\nabla}\right)^{-1}$; thus, the two Berry phases cancel. These are the results listed in Table V.

\section{COMMENTS ON GAUGE FLUCTUATIONS}

So far, most of our calculations, especially those related to spinon band topology, have been done in the mean-field framework. We now discuss in more detail why such calculations are expected to capture the correct monopole quantum numbers in a strongly interacting spin-liquid system. Most of the discussions below are not new and are included mainly for pedagogical purposes.
What a parton construction [like Eq. (2)] really provides is a mapping from a spin system to a lattice gauge theory (details of the mapping can be found in, e.g., Refs. [4,5]). The two systems share the same physical Hilbert space, even though a simple Hamiltonian in one system may correspond to a complicated (but still local) one in the other system. As we explore the space of local Hamiltonians that preserve the relevant global symmetries, the two systems should produce the same phase diagram. In other words, phases that can be realized in the lattice gauge theory should also be realizable in the spin system - even though the corresponding spin Hamiltonian could be complicated and hard to find.

In the lattice gauge theory, the continuum $\mathrm{QED}_{3}$ [Eq. (4)] can be obtained in a controlled manner through standard reasoning: Starting from a mean-field fermion Hamiltonian [Eq. (3)] that produces the Dirac fermions in the IR, we weakly couple the fermions to a lattice $U(1)$ gauge field. We can then safely take the continuum limit in this weak coupling regime and obtain the continuum $\mathrm{QED}_{3}$ Lagrangian, Eq. (4). We define $U(1)$ Dirac spin liquids as the phase of spin systems that is equivalent (or smoothly connected) to the continuum $\mathrm{QED}_{3}$ obtained from the lattice gauge theory side in a controlled manner. In the language of the renormalization group, the weakly coupled lattice gauge theory flows to a fixed point (in general, strongly coupled) in the IR, and we define the corresponding Dirac spin liquid as any spin system (in general, strongly interacting) that flows to the same fixed point in the IR. This line of thinking is 
nothing new: It is the same logic that underlies the mapping from the lattice Ising model to the continuum $\phi^{4}$ theory (the role of gauge coupling in our case is played by nonlinear couplings of the $\phi$ field). In the Ising model case, this discussion is very often kept implicit because of the simplicity of the phase diagram. For phases with emergent gauge fields, there are often many competing phases, which makes the phase diagram complicated; thus, the issue of the continuum limit deserves a more careful discussion.

In the continuum theory, the coupling constant $1 / e^{2}$ provides a length scale (much larger than the lattice scale), and only above this scale does the gauge fluctuation become strong and require careful consideration. This result immediately implies that the UV contributions to monopole quantum numbers [the $U(1)_{\text {top }}$ phase factors in the symmetry actions] can be obtained through a meanfield calculation since on the lattice scale the system is essentially free. The weak coupling between the fermions and gauge fields in the UV can still modify certain aspects perturbatively, but since the $U(1)_{\text {top }}$ phase factors take discrete values in our case, they cannot receive any perturbative correction. To even better control the calculation, we also consider massive Dirac fermions (for example, with quantum spin Hall band gaps) with $m \gg e^{2}$. In this case, the gauge coupling remains weak from UV to IR, so a mean-field calculation will certainly suffice. The $m \rightarrow 0$ limit is more subtle since gauge fluctuation eventually becomes strong. In this case, we made the assumption that the leading (most relevant) monopoles are those in the lowest representation of the flavor symmetry group [vector of $S O(6)$ ], and one of these leading monopoles becomes the unique monopole in the $m \gg e^{2}$ limit. This assumption can be rigorously justified only in the large $N_{f}$ limit, but it is physically reasonable even at $N_{f}=4$ as long as the IR fixed point actually exists (i.e., without spontaneous chiral symmetry breaking). At this IR fixed point, monopoles are included as part of the critical fluctuations and are crucial for the strongly coupled dynamics. However, its symmetry properties were decided from the UV, which can be weakly coupled and therefore theoretically tractable.

There is another closely related but distinct way to view the parton approach in the literature known as the Gutzwiller projection. In this approach, Eqs. (2) and (3) are viewed as machinery to generate trial wave functions for the spin systems: One takes the ground-state wave functions of the spinon mean-field Hamiltonian, Eq. (3) (a Slater determinant), and projects to the physical Hilbert space with one spinon per site. This approach has the advantage of being explicit in every step, and in some cases, it is also connected to other approaches for strongly correlated systems such as tensor networks. The Gutzwiller projection also provides extremely valuable intuitions and, in many cases, leads to wave functions that faithfully describe certain quantum spin liquids. However, the projection is, in general, not theoretically controlled-in the gauge theory language, it corresponds to a lattice gauge theory at strong coupling. For this reason, the projected wave function is, in general, not guaranteed to faithfully describe the spin-liquid phase (defined as the IR phase of a UV weakly coupled gauge theory). The most wellknown example may be the Laughlin fractional quantum Hall states at small filling fractions: A Laughlin state at filling fraction $\nu=1 / k$ ( $k$ positive integer) can be thought of as a parton state through $c=f_{1} f_{2}, \ldots, f_{k}$, where each $f_{i}$ fills a lowest Landau level. This case motivates emergent Chern-Simons gauge theories that eventually describe nontrivial topological orders in the fractional quantum Hall regime. For arbitrarily large $k$, the gauge theories remain well defined and can certainly be realized as the ground states of some local (albeit complicated) Hamiltonians. However, the pristine Laughlin wave functions at large enough $k$ (numerically about 70) actually describe Wigner crystals [61]. The lesson is that to understand quantum phases of matter, we should focus on universal aspects of the physics (such as those described by continuum field theories) rather than particular wave functions.

\section{CONSEQUENCES OF MONOPOLES: SOME EXAMPLES}

The symmetries of monopole operators crucially constrain how monopoles enter the theory and the phases to which DSL ultimately flows or is in proximity. In summary, on bipartite lattices, monopoles confine the theory and lead to ordered phases; however, for triangular or kagome lattices, DSL could be stable, and the neighboring symmetry-breaking phases have distinctive features associated with monopole quantum numbers. Monopole operators, as order parameters, require that the associated ordered phases have the same symmetry group as respective monopoles. In a parallel paper [28], we report in detail the physical consequences of monopole quantum numbers, and below, we discuss some of the most outstanding results on square and triangular lattices.

\section{A. Square lattice}

As shown in Table II, $\operatorname{Im}\left[\Phi_{2}\right]$ stays invariant under all symmetries and is allowed in the Lagrangian. The $S O(5)$ subgroup of $S O(6)$ in which the remaining five nontrivial monopoles transform suffices to accommodate all physical symmetries (see Sec. IVA). This relevant monopole operator will proliferate to confine gauge fluctuations, and meanwhile, a fermion mass develops to gap the matter fields. In particular, this fermion mass is more likely to be one of the five masses that transform in a vector representation of the $S O(5)$ like the other five monopoles [recall that 15 fermion masses form an adjoint of $S O(6)$ and are 
split into $5 \oplus 10$ of its $S O(5)$ subgroup] now that a symmetry allowed term

$$
\sum_{i=1,2,3} \bar{\psi} \tau^{2} \sigma^{i} \psi \operatorname{Re}\left[\Phi^{i+3}\right]+\bar{\psi} \tau^{1} \psi \operatorname{Re}\left[\Phi^{3}\right]-\bar{\psi} \tau^{3} \psi \operatorname{Re}\left[\Phi^{1}\right]
$$

is present. This bilinear coupling between fermion mass and monopole means that they are equivalent under physical symmetry transformations. Once a fermion mass condenses, the resulting order can be described either by the mass or by the monopole it couples to alone. Indeed, among the five masses, $\bar{\psi} \tau^{2} \sigma^{i} \psi$ transform as the Néel order parameters, and $\bar{\psi} \tau^{1,3} \psi$ transform as the two columnar VBS order parameters, respectively (Table VII). Henceforth, the DSL describes a Néel or VBS ordered phase on the square lattices because of the presence of a trivial monopole. By fine-tuning to the point where no fermion masses are generated, one accesses the deconfined quantum critical point with $S O(5)$ symmetry.

Before ending this section, a comment is in order. There is traditional folklore that on nonbipartite lattices, colinear (Néel) antiferromagnetic orders are frustrated, and therefore it becomes easier, compared to that on bipartite lattices, to quantum fluctuate the magnetic order and obtain a spin liquid. The bipartite-nonbipartite dichotomy discussed in our work, namely, that DSL is less likely to be realizable on bipartite lattices, is very different from this folklore. In fact, monopoles in DSLs that behave as magnetic orders (spin triplets) behave similarly on bipartite and nonbipartite lattices. What made the difference was the spin-singlet monopoles. On nonbipartite lattices, the spin-singlet monopoles behave as some complicated types of VBS order parameters and are all nontrivial. On bipartite lattices, however, the VBS orders are too simple to occupy all three

TABLE VII. The transformation of fermion bilinears $M_{i j} \equiv \bar{\psi} \sigma^{i} \tau^{j} \psi(i, j=0,1,2,3)$ on four types of lattices (staggered flux mean field on the square lattice). Note that $T_{1 / 2}$ and reflection are marked in Fig. 6 rotation denotes fourfold rotation around the site for the square lattice and sixfold rotation for the honeycomb, kagome, and triangular lattices.

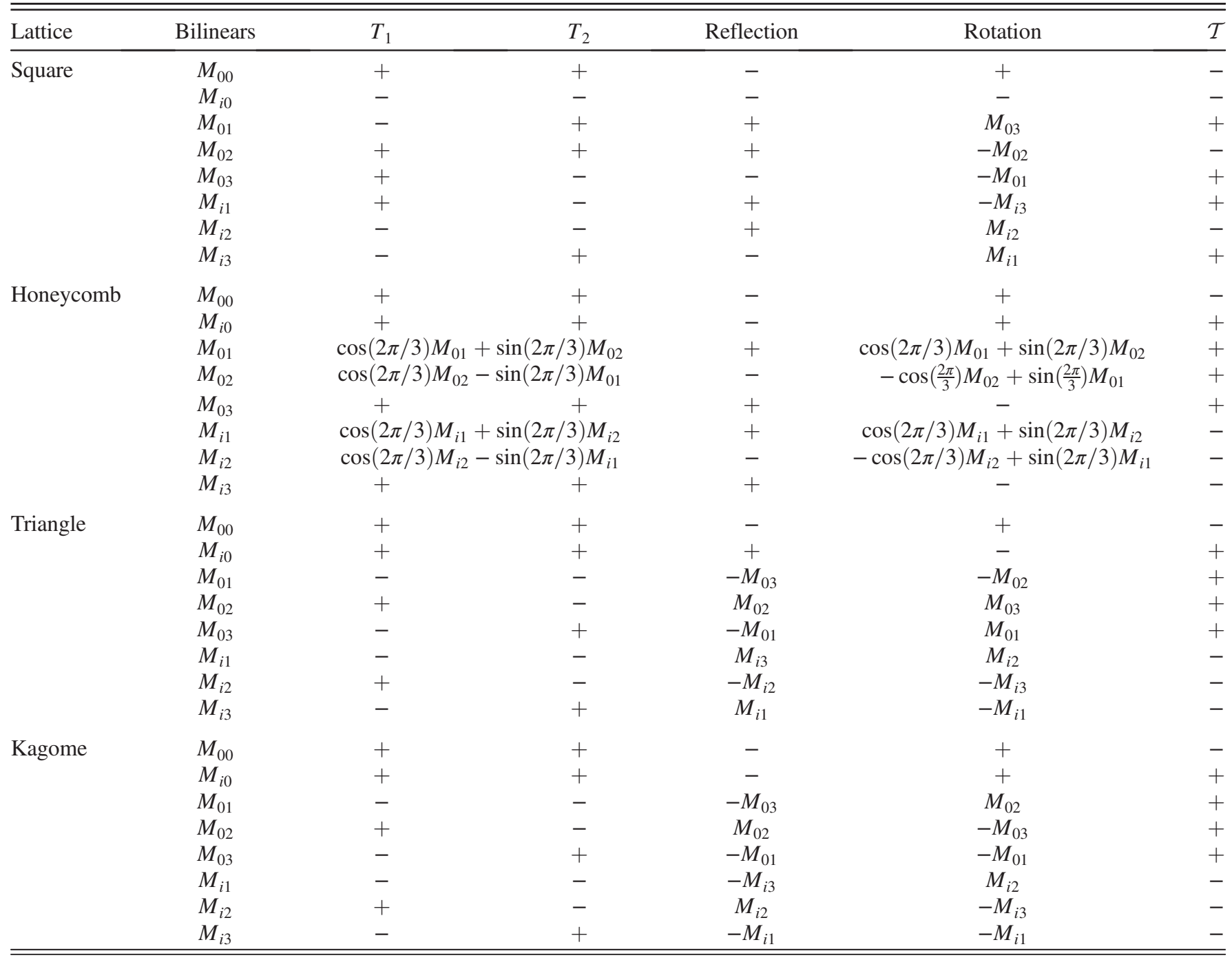


singlet monopoles $\left(\Phi_{1,2,3}\right)$, which leads to one of the singlet monopoles being completely trivial. Therefore, in contrast to traditional wisdom, in the DSL case it is the complexity of VBS order parameters that made the difference between bipartite and nonbipartite lattices.

\section{B. Triangular lattice}

As shown in Sec. VI A, a $\pm(2 \pi / 3)$ Berry phase attaches to monopoles under translations. This peculiar phase forbids either an elementary monopole or a double-strength monopole; i.e., the leading-order monopole allowed on the triangular lattice is a threefold monopole formally written as $\Phi_{1} \Phi_{2} \Phi_{3}+$ H.c., which is highly likely irrelevant in the renormalization group sense. The DSL can be a stable phase.

Moreover, one considers its proximate symmetrybreaking phases accessed by tuning interactions to drive chiral symmetry breaking [28]. The discussion assumes first a condensed fermion mass and then an energetically favored monopole proliferation. For example, if a quantum spin Hall mass, $\bar{\psi} \sigma^{3} \psi$, condenses and gaps the fermions, this lowers the energy of two zero modes that carry $S_{z}=$ $-1 / 2$ and selects the monopole associated with these two zero modes filled as the lowest-energy one. This monopole is $f_{1, \downarrow}^{\dagger} f_{2, \downarrow}^{\dagger} \mathcal{M}_{\text {bare }}=\Phi_{4}^{\dagger}+i \Phi_{5}^{\dagger}$ according to Eq. (8). Since $\left\{\Phi_{4}, \Phi_{5}, \Phi_{6}\right\}$ transform as $\left\{S_{x}, S_{y}, S_{z}\right\}$ spin components in the $S O(3)_{\text {spin }}$ group, $\Phi_{4}+i \Phi_{5}$ is equivalent to spin operators $S_{x}+i S_{y}$. Under translations $T_{1,2}, \Phi_{4}^{\dagger}+i \Phi_{5}^{\dagger}$ acquires a phase $\pm(2 \pi / 3)$, respectively; in other words, the order parameter $S_{x}+i S_{y}$ rotates by $\pm 2 \pi / 3$ upon translations. Intuitively, $S_{x}+i S_{y}$ characterizes the ordered spin moments in the $x y$ plane where the angle of complex $S_{x}+i S_{y}$ tells the direction of ordered moments. This translation Berry phase hence translates to the angle between neighboring $x y$ plane spin moments. Hence, the quantum spin Hall mass drives the familiar $120^{\circ}$ magnetic order on the triangular lattices. We note that this Berry phase effect, which is crucial to the identification of final orders, is unique to monopole operators. This result also provides useful guidance to identify a DSL in spectroscopic measurements or numerical studies. As shown in Fig. 8, monopoles carry momenta like $(2 \pi / 3,-2 \pi / 3),(\pi / 3,2 \pi / 3)$, while fermion bilinears (Table VII) are located at $\Gamma, M$ points in the Brillouin zone.

Hence, our results on monopole quantum numbers not only solve the longstanding question on the stability of DSL but also unveil the nature of its neighboring symmetry-breaking orders. When stabilized, it is a promising candidate theory in numerics [62] and for real materials [63], with distinctive features given by monopoles' unique (angular) momenta or other quantum numbers [28]; even if unstable on bipartite lattices, DSL organizes the plethora of ordered phases a host organizing the plethora of ordered phase.

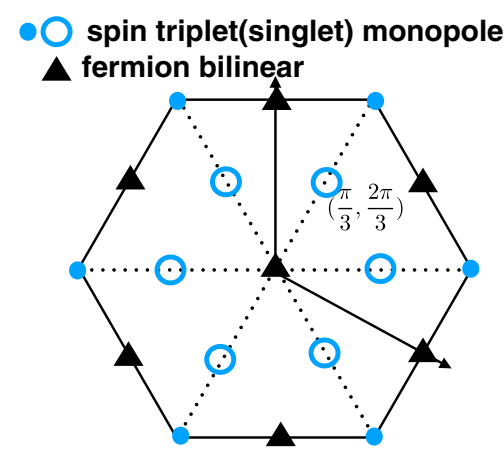

FIG. 8. The momenta of monopoles and fermion bilinears in the triangular lattice DSL-signatures of a DSL system in experiments such as neutron scattering. Solid or empty circles represent momenta of spin-triplet or spin-singlet monopoles, distinct from all fermion bilinear operators, and black triangles represent those of fermion bilinears.

\section{ANOMALIES AND LIEB-SCHULTZ-MATTIS}

The celebrated Lieb-Schultz-Mattis (LSM) type of theorems [41-43] strongly constrain the possible lowenergy theories that can emerge from a lattice system. For example, a trivially gapped ground state (without topological orders or symmetry breaking) cannot emerge out of a system with spin- $1 / 2$ per lattice unit cell with translation symmetry. In the IR limit, these constraints are manifested as symmetry anomalies in the continuum field theory descriptions [44-47].

In the $C P^{1}$ (slave boson) representation of spin- $1 / 2$ systems on the square lattice, the monopole quantum numbers were fixed by LSM constraints [45]. Essentially, the LSM theorem requires certain symmetry anomalies in the effective $C P^{1}$ field theory, which can be matched only if the monopole carries the right quantum number, e.g., \pm 1 angular momentum under $C_{4}$. It is then natural to ask to what extent the monopole quantum numbers in Dirac spin liquids are determined by LSManomaly constraints. In this section, we show that monopole quantum numbers associated with $\mathbb{Z}_{2}$ symmetries (such as inversion) are indeed determined by LSM-anomaly constraints in DSL, while those associated with $\mathbb{Z}_{3}$ symmetries (such as $C_{3}$ ) are not.

Let us start from the $\mathrm{QED}_{3}$ theory and try to gauge the $S O(3)_{\text {spin }} \times S O(3)_{\text {valley }} \times U(1)_{\text {top }}$ symmetry [64], as we will be interested in those microscopic symmetries that can be embedded into this group. The anomaly associated with these symmetries can be calculated. One way to interpret the anomaly is to imagine a $(3+1) d$ SPT state that hosts the $\mathrm{QED}_{3}$ theory on its boundary, and the bulk SPT is characterized by a response theory

$$
S_{\text {bulk }}=i \pi \int_{X_{4}}\left[w_{2}^{s} \cup w_{2}^{v}+\left(w_{2}^{s}+w_{2}^{v}+\frac{d A_{\text {top }}}{2 \pi}\right) \cup \frac{d A_{\text {top }}}{2 \pi}\right] \text {, }
$$


where $w_{2}^{s, v}$ are the second Stiefel-Whitney classes of the $S O(3)_{\text {spin/valley }}$ bundles, respectively, and $A_{\text {top }}$ is the $U(1)$ gauge field that couples to the $U(1)_{\text {top }}$ charge. We outline the derivation of the above expression (which is very similar to that in Ref. [65]) in Appendix B. The first term is essentially a descendant of the parity anomaly of Dirac fermions, and the terms involving $A_{\text {top }}$ simply represent the fact that an $A_{\text {top }}$ monopole (not to be confused with the $a$ monopole discussed throughout this paper) carries halfspin under $S O(3)_{\text {spin/valley }}$ and is a fermion-this is because a bare $A_{\text {top }}$ monopole carries a gauge charge under $a$, which must be compensated by attaching a spinon $\psi$ field in the original $\mathrm{QED}_{3}$ to make it local, and this process also attaches the nontrivial quantum numbers of $\psi$ to the $A_{\text {top }}$ monopole.

Our remaining task is simply to embed the lattice symmetries into the $S O(3)_{\text {valley }} \times U(1)_{\text {top }}$ group and to see if the correct anomaly from LSM is reproduced. The PSG determines the $S O(3)_{\text {valley }}$ part of the lattice symmetries, which in turn determines $w_{2}^{v}$. The only unknown is the relation between the lattice symmetries and $A_{\text {top }}$, determined by the $U(1)_{\text {top }}$ Berry phase in the symmetry realizations.

Let us first consider $\mathbb{Z}_{2}$ inversion symmetries. On the triangular lattice, these symmetries are not meaningful since they involve charge conjugation. We consider the other three lattices in detail.

On the square lattice, the site-centered inversion involves a nontrivial $S O(3)_{\text {valley }}$ rotation $\psi \rightarrow i \tau^{2} \psi$. So if we gauge the inversion symmetry (called the $\mathbb{Z}_{2}$ connection $\gamma$ ), the $\pi w_{2}^{s} w_{2}^{v}$ term in Eq. (56) becomes $\pi w_{2}^{s} \gamma^{2}$ (shorthand for $\gamma \cup \gamma$ ), which is exactly the anomaly imposed by LSM (simply reflecting the fact that there is a spin-1/2 Hilbert space at the inversion center on the lattice). Therefore, the other terms in Eq. (56) should not contribute further anomalies. This means that $d A_{\text {top }}=0$; i.e., there is no additional $U(1)_{\text {top }}$ phase factor associated with inversion. This case is indeed what we have in Table II, where inversion only implements the $S O(3)_{\text {valley }}$ rotation by $\Phi_{1,3} \rightarrow-\Phi_{1,3}$.

On the honeycomb lattice, the $\pi w_{2}^{s} w_{2}^{v}$ term likewise gives a $\pi w_{2}^{s} \gamma^{2}$ anomaly, where $\gamma$ is again the inversion gauge field. However, since on the lattice there is no spin at the inversion center, there should be no actual anomaly. This means that the other terms in Eq. (56) should contribute another $\pi w_{2}^{s} \gamma^{2}$ term to the anomaly, which can be done by having $d A_{\text {top }}=2 \pi \gamma^{2}$. Thus, under inversion, the monopole should pick up an additional (-1) phase, exactly in accordance with Table I.

On the kagome lattice, the hexagon-centered inversion does not involve any nontrivial $S O(3)_{\text {valley }}$ rotation for the Dirac fermions; therefore, the $\pi w_{2}^{s} w_{2}^{v}$ term does not contribute an anomaly for inversion. The LSM constraint also requires no anomaly since on the lattice there is no spin at the inversion center. Therefore, the terms involving $A_{\text {top }}$ in Eq. (56) should not give rise to any anomaly either. Thus, under inversion, the monopoles stay invariant, again in accordance with Table V.

We now consider $\mathbb{Z}_{3}$ (or any $\mathbb{Z}_{2 k+1}$ ) symmetries like the $C_{3}$ rotations. In this case, the anomalies become trivial no matter how we embed the symmetry to $S O(3)_{\text {valley }} \times U(1)_{\text {top. }}$. This case can be seen most easily by writing the anomalies involving $S O(3)_{\text {spin }}$ as $\pi w_{2}^{S} w_{2}^{S O(3)_{\text {valley }} \times U(1)_{\text {top }}}$ [recall that $\left.w_{2}^{S O(2)}=d A / 2 \pi(\bmod 2)\right]$ and using the fact that $w_{2}=0$ for a $\mathbb{Z}_{3}$ bundle. Therefore, the anomaly-based argument does not say anything about monopole quantum numbers for these symmetries.

As a final example, let us consider translation symmetries $T_{1,2}$ on the triangular lattice. These symmetries act as $\mathbb{Z}_{2} \times \mathbb{Z}_{2}$ on Dirac fermions but could also involve a $\mathbb{Z}_{3}$ subgroup of $U(1)_{\text {top }}$ [one can show that translation involves the Berry phase $2 n \pi / 3(n \in \mathbb{Z})$ from algebraic relations of the space group [28] ]. The $\mathbb{Z}_{2} \times \mathbb{Z}_{2}$ part gives an anomaly $\pi w_{2}^{s} x y$, where $x, y$ are the $\mathbb{Z}_{2}$ forms associated with $T_{1,2}$, and this is exactly the LSM anomaly since we have one spin- $1 / 2$ per unit cell. The $\mathbb{Z}_{3}$ part, however, will not further modify the anomaly, so the two inequivalent choices of Berry phase ( $e^{i 2 \pi / 3}$ or trivial) are both allowed by LSM.

\section{THREE DIMENSIONS: MONOPOLE PSG FROM CHARGE CENTERS}

The charge (Wannier) center approach can also be generalized to three dimensions. Consider a 3D $U(1)$ quantum spin liquid with gapped spinons (charge) and magnetic monopoles. Recall that in $3 \mathrm{D}$ a monopole is a point excitation, and the monopole creation operator is a nonlocal operator. Such spin liquids have been extensively discussed in the context of quantum spin ice materials [2]. The nonlocal nature of monopoles in 3D implies that unlike the $2 \mathrm{D}$ case, they can transform projectively under physical symmetries like the spinons. The relevant question then is how the spinon band topology (or SPT-ness) affects the monopole projective symmetry quantum number (or simply monopole PSG).

We now show that the monopole PSGs associated with lattice rotation symmetries are determined by the effective gauge charges sitting at the rotation centers, in cases when the rotation symmetries do not involve charge conjugation (namely, when the monopole flux is invariant under the lattice rotations). To see this, first consider a single charge, or any odd-integer charge, in space with the full $S O(3)$ rotation symmetry around the charge. By examining the Aharonov-Bohm phase for a monopole moving around the charge, one concludes that the monopole carries halfinteger angular momentum under the space $S O(3)$ rotation [66]; i.e., the monopole transforms projectively under 
$S O(3)$. Now on a lattice, the $S O(3)$ is broken down to a discrete subgroup, but as long as the remaining rotation group $G$ admits a projective representation $\omega_{2} \in$ $H^{2}(G, U(1))$ that is a descendant of the spin-1/2 representation when $G$ is embedded into $S O(3)$, the monopole will transform projectively under $G$ according to $\omega_{2}$.

The simplest example is the dihedral group $D_{2}=$ $\mathbb{Z}_{2} \times \mathbb{Z}_{2}$, corresponding to $\pi$ rotations about three orthogonal axes. If an odd number of gauge charges effectively sit at the rotation center, the monopole will transform under $D_{2}$ such that different $\mathbb{Z}_{2}$ rotations anticommute.

\section{DISCUSSION AND FUTURE DIRECTIONS}

We have demonstrated a surprising mapping between Landau order parameters and the symmetry-protected band topology of fermions. The link is established by studying the properties of magnetic monopoles in a Dirac spin liquid. Knowledge of the spinon band topology allows us to analytically calculate monopole symmetry quantum numbers. This in turn allows us to identify the set of order parameters that are enhanced in the vicinity of a Dirac spin liquid. Thus, results involving gapped and noninteracting fermions, which is an analytically well-controlled limit, are used to extract key information about a strongly interacting and gapless system, the Dirac spin liquid. This treatment can be readily generalized to systems with higher spins and/ or more Dirac fermions. We also showed that on bipartite lattices, there always exists a symmetry-trivial monopole due to the existence of a parent $S U(2)$ gauge theory. In a separate publication, we have discussed the physical consequence of the monopole properties as well as signatures of a Dirac spin liquid, which can be accessed in numerics and in scattering experiments on candidate materials [28].

Even though all our calculations of the Wannier centers were done in the free-fermion framework, notions like the monopole quantum numbers are certainly well defined beyond free fermions. In fact, the connection between Wannier centers and monopole quantum numbers gives intrinsically interacting definitions of notions like Wannier centers and fragile topology (in accordance with the recent work $[67,68])$.

The monopole angular momenta arising from charge (Wannier) centers are consistent with another simple fact in lattice gauge theory: In the strong-coupling limit of a lattice gauge theory (which is fully confined), all the gauge charges simply sit on the defining sites of the lattice and do not fluctuate. Therefore, if the gapped charge fields have their effective charge centers (Wannier centers) off the sites (say, at plaquette centers), the strong-coupling limit must destroy such a state and pull the charges back to the lattice sites, implying that the lattice symmetries (that prevent charges from moving away from nontrivial centers) must be broken along the way.
One can use this as an intuitive way to understand the following well-known statement: The $S U(2)$ Yang-Mills theory in $(3+1)$ dimensions with $\theta=\pi$ cannot be confined to a trivial phase without breaking time-reversal symmetry (for a recent exposure, see Ref. [69]). When put on the lattice, $\theta=\pi$ can be generated by introducing fundamental fermions and putting the fermions into a topological superconductor state [protected by $S U(2) \times \mathcal{T}$ ]. By definition, the topological superconductor cannot be deformed (without closing the gap or breaking symmetries) to a trivial product state with local $S U(2)$ singlets on lattice sites-but this is exactly what the strong-coupling limit demands. Therefore, if the theory indeed flows to infinite coupling, without closing the fermion gap, then time-reversal symmetry must be broken in the IR.

We end with a discussion of open issues:

(i) It is also straightforward to apply the charge center method to $\mathbb{Z}_{2}$ spin liquids (or other discrete gauge theories) in $(2+1) d$. For example, if a nontrivial $\mathbb{Z}_{2}$ charge (effectively) sits at the center of a plaquette, then the gauge flux (vison) will transform projectively under the lattice rotation around the plaquette center. This situation is similar to the one for 3D $U(1)$ spin liquids discussed in Sec. X.

(ii) One natural question is as follows: Given a microscopic Hilbert space (say, one spin-1/2 per site on the triangular lattice), is it possible to realize a different $U(1)$ Dirac spin liquid, with the same field content and symmetry realizations (PSG) but different monopole quantum number from the one discussed in this work? For example, is there a spinon mean-field ansatz that gives identical Dirac dispersion and PSG but with monopoles at different momenta, say, $T_{1,2}: \Phi_{4 / 5 / 6} \rightarrow \Phi_{4 / 5 / 6}$ (as opposed to that in Table III)? Unlike the bosonic spinon theory for deconfined criticality, some of the monopole quantum numbers in our examples are not linked to LSM constraints [45], as discussed in Sec. IX. So an alternative $U(1)$ Dirac spin liquid (with the same PSG but different monopole quantum numbers) seems to be allowed on general grounds-finding a concrete example is left for future work.

(iii) In Secs. V and VI, we calculated the angular momenta of monopoles using charge centers and then obtained their momenta by composing different rotations. In the absence of lattice rotation symmetries, the angular momenta are not defined, but the momentum of a monopole is still well defined (although it may not be quantized). It is then natural to ask what determines monopole momentum when lattice rotation symmetries are not considered. Recall that with rotation symmetries, the momentum is given by the difference between angular momenta around different rotation centers, which is in turn given by the difference between gauge charges 
sitting at different rotation centers. The latter appears to be nothing but the dipole moment in the unit cell, which in the more familiar language is just the polarization density. In fact, by comparing Fig. 6 and the monopole momenta for each spinon insulator, we can infer that monopole momentum $\mathbf{k}_{\mathcal{M}}$ is given by the polarization density $\mathbf{P}$ (dipole density per unit cell) through $\mathbf{k}_{\mathcal{M}}=2 \pi \hat{z} \times \mathbf{P}$. In a forthcoming work, we will develop the connection between polarization and monopole momentum in detail without assuming lattice rotation symmetries [28].

\section{ACKNOWLEDGMENTS}

We gratefully acknowledge helpful discussions with Chao-Ming Jian, Eslam Khalaf, Shang Liu, Jacob McNamara, Max Metlitski, Adrian H. C. Po, Ying Ran, Subir Sachdev, Cenke Xu, Yi-Zhuang You, and Liujun Zou. X.-Y.S. acknowledges the hospitality of the Kavli Institute of Theoretical Physics (NSF PHY-1748958). Y.-C. H. was supported by the Gordon and Betty Moore Foundation under the EPiQS initiative, GBMF4306, at Harvard University. A. V. was supported by a Simons Investigator award. C. W. was supported by the Harvard Society of Fellows. Research at Perimeter Institute (Y.-C. H. and C. W.) was supported by the Government of Canada through the Department of Innovation, Science and Economic Development Canada and by the Province of Ontario through the Ministry of Research, Innovation and Science.

Note added.-In a different parallel work [68], monopole quantum numbers were used as a probe of free-fermion band topology for a specific class of models. Here, however, monopoles are dynamical objects, and the underlying models are of strongly interacting quantum magnets.

\section{APPENDIX A: MEAN FIELD, PROJECTIVE SYMMETRY GROUP, AND FERMION BILINEAR TRANSFORMATIONS}

\section{Square}

We adopt a mean field where $t_{i j}=(-1)^{y}$ for horizontal links and $t_{i j}=1$ for vertical links on the square lattice, which creates $\pi$ flux on every plaquette. This choice enlarges the unit cell to contain two sites (sublattices $A, B)$ with a vertical link. There are two gapless points in the reduced Brillouin zone at $\mathbf{Q}=(\pi / 2, \pi), \mathbf{Q}^{\prime}=-\mathbf{Q}$.

The projective symmetry group reads (applicable to the staggered flux state)

$$
\begin{aligned}
& T_{1}: \psi \rightarrow i \mu^{3} \sigma^{2} \tau^{3} \psi^{*}, \quad T_{2}: \psi \rightarrow i \mu^{3} \sigma^{2} \tau^{1} \psi^{*}, \\
& R_{x}: \psi \rightarrow \tau^{3} \mu^{3} \psi, \quad C_{4}: \psi \rightarrow \frac{1}{\sqrt{2}} \mu^{3} \sigma^{2} e^{-i(\pi / 4) \tau^{2}} e^{i(\pi / 4) \mu^{1}} \psi^{*}, \\
& \mathcal{T}: \psi \rightarrow \tau^{2} \mu^{1} \psi^{*},
\end{aligned}
$$

$$
\mathcal{C}: \psi \rightarrow i \mu^{3} \sigma^{2} \psi^{*}
$$

where $\mathcal{C}$ denotes charge conjugation that reverses the flux $\phi \rightarrow-\phi$. Note that the projective representation is different from that in the main text (Sec. IVA) because of the case for $\pi$ flux, where for $T_{1,2}, C_{4}, \mathcal{T}$, one does not need additional charge conjugation $\mathcal{C}$; i.e., the $T_{1,2}, C_{4}, \mathcal{T}$ above are identified as $\mathcal{C} T_{1,2}, \mathcal{C} C_{4}, \mathcal{C} \mathcal{T}$ in the main text.

\section{Honeycomb lattice}

On the honeycomb lattice, with the mean-field ansatz of uniform fermion hopping, one could similarly work out the PSG and the constraints on monopole quantum numbers. The Dirac points stay at momenta $\mathbf{Q}=[(2 \pi / 3),(2 \pi / 3)]$, $\mathbf{Q}^{\prime}=-\mathbf{Q}$.

The physical symmetries act as

$$
\begin{aligned}
T_{1 / 2}: \psi & \rightarrow e^{-i(2 \pi / 3) \tau^{3}} \psi, \\
C_{6}: \psi & \rightarrow-i e^{-i(\pi / 6) \mu^{3}} \tau^{1} e^{-i(2 \pi / 3) \tau^{3}} \psi, \quad R_{x}: \psi \rightarrow-\mu^{2} \tau^{2} \psi, \\
\mathcal{T}: \psi & \rightarrow-i \sigma^{2} \mu^{2} \tau^{2} \psi, \quad \text { charge conjugation } \\
C: \psi & \rightarrow i \mu^{1} \tau^{1} \sigma^{2} \psi^{*},
\end{aligned}
$$

where $T_{1 / 2}$ is the translation along two basis vectors with $2 \pi / 3$ angle between them, $C_{6}$ is $\pi / 3$ rotation around the center of a honeycomb plaquette, and $R_{x}$ denotes reflection along the direction of the unit cell (Fig. 3).

From the transformation of Dirac fermions, one gets the transformation of fermion masses as in Table VII.

\section{Triangular lattice}

There is a "staggered $\pi$-flux" configuration of $t_{i j}$ on the triangular lattice. We choose a particular gauge of $t_{i j}$ to realize this mean field as in Fig. 9. Under an appropriate basis, the low-energy Hamiltonian reads as the standard form with four gapless Dirac fermions.

For later purposes, the charge conjugate operation is given here as

$$
\psi \rightarrow W_{c} \psi^{*}=\frac{1}{\sqrt{3}}\left(-i I_{4 \times 4}-\mu^{3}+\mu^{1}\right) \psi^{*} .
$$

The PSGs for all the symmetry operations are the following:

$\psi \stackrel{T_{2}}{\rightarrow} i \tau^{3} \psi, \quad \psi \stackrel{T_{1}}{\rightarrow}-i \tau^{2} \psi, \quad \psi \stackrel{\mathcal{T}}{\rightarrow} i \sigma^{2} \mu^{2} \tau^{2} K \psi(-k)$,

$$
\begin{aligned}
& \psi\left(k_{1}, k_{2}\right) \stackrel{C_{6}}{\rightarrow} i \sigma^{2} W_{C_{6}} \psi^{\dagger}\left(-\frac{k_{2}}{2}, 2 k_{1}-k_{2}\right), \\
& \psi\left(k_{1}, k_{2}\right) \stackrel{R}{\rightarrow} i \sigma^{2} W_{R} \psi^{\dagger}\left(k_{1}-\frac{k_{2}}{2},-k_{2}\right),
\end{aligned}
$$



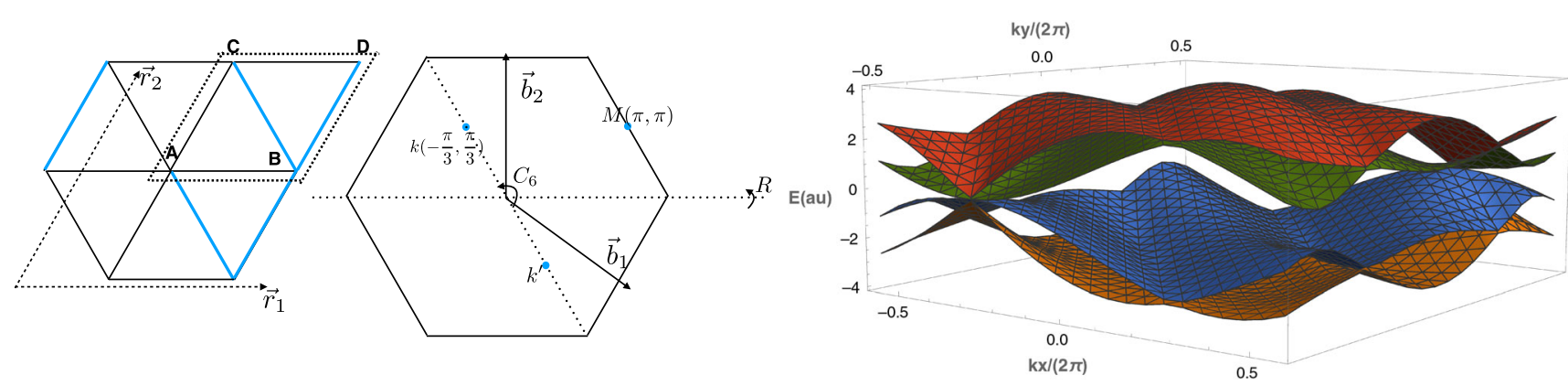

FIG. 9. Left panel: The gauge choice (blue or black bonds denote negative or positive hopping strength with equal amplitudes) and the Brillouin zone with the $M$ point the gapless point and $k, k^{\prime}$ staying invariant under PSG of threefold rotations. Right panel: The energy spectrum of the staggered flux spinon model on the triangular lattice in the reduced Brillouin zone with a four-site unit cell. The spectrum is gapless at momentum $(\pi, \pi)$.

where

$$
\begin{aligned}
W_{C_{6}} & \left.=e^{-i \gamma^{3}(\pi / 6)} W_{c} e^{e} i \frac{\pi}{3} \tau^{C}\right] \quad \tau^{C}=\frac{1}{\sqrt{3}}\left(\tau^{1}+\tau^{2}+\tau^{3}\right), \\
W_{R} & =\frac{\left(\gamma^{1}-\sqrt{3} \gamma^{2}\right)}{2} W_{c} \frac{\tau^{3}-\tau^{1}}{\sqrt{2}} .
\end{aligned}
$$

\section{Kagome lattice}

On the kagome lattice, similar to the triangular case, Hermele et al. calculated the kagome DSL with a staggered flux mean-field ansatz, with three gamma matrices as $\gamma_{\nu}=\left(\mu^{3}, \mu^{2},-\mu^{1}\right)$, and we have, for the PSG of Dirac fermions,

$$
\begin{aligned}
& T_{1}: \psi \rightarrow\left(i \tau^{2}\right) \psi \quad T_{2}: \psi \rightarrow\left(i \tau^{3}\right) \psi, \\
& R_{y}: \psi \rightarrow\left(i \mu^{1}\right) \exp \left(\frac{i \pi}{2} \tau_{r y}\right) \psi, \\
& C_{6}: \psi \rightarrow \exp \left(\frac{i \pi}{3} \mu^{3}\right) \exp \left(\frac{2 \pi i}{3} \tau_{R}\right) \psi, \\
& \mathcal{T}: \psi \rightarrow\left(i \sigma^{2}\right)\left(i \mu^{2}\right)\left(-i \tau^{2}\right) \psi,
\end{aligned}
$$

where

$$
\tau_{r y}=\frac{-1}{\sqrt{2}}\left(\tau^{1}+\tau^{3}\right), \quad \tau_{R}=\frac{1}{\sqrt{3}}\left(\tau^{1}+\tau^{2}-\tau^{3}\right) .
$$

\section{APPENDIX B: THE $S O(3)_{s} \times S O(3)_{v} \times U(1)_{\text {top }}$ ANOMALY}

We take the $\mathrm{QED}_{3}$ Lagrangian and gauge the $S O(3)_{s} \times$ $S O(3)_{v} \times U(1)_{\text {top }}$, with gauge connections denoted by $\mathcal{A}_{s}, \mathcal{A}_{v}, A_{\text {top }}$, respectively. We also regularize the Dirac fermions using a Pauli-Villars regulator (which is different from the notation used in the main text) and define the Euclidean partition function of a Dirac fermion coupled with a gauge field $A$ and metric $g$ to be

$$
Z[A, g]_{P V}=|Z[A, g]| \exp \left(-\frac{i \pi}{2} \eta[A, g]\right)
$$

where $\eta[A, g]$ is the $\eta$ invariant, which is similar to a halflevel Chern-Simons term classically but is gauge invariant unlike the half-level Chern-Simons term (see Ref. [53] for more details). One can simply interpret the $\eta$ invariant as coming from an additional gapped Dirac fermion. Now, the gauged $\mathrm{QED}_{3}$ theory should be properly written as

$$
\begin{aligned}
S= & \int\left[\left(\bar{\psi} \not D_{a / 2, A_{s}, A_{v}, g} \psi\right)_{P V}+\frac{i}{2} C S[a]+\frac{i}{2} C S\left[A_{s}\right]\right. \\
& \left.+\frac{i}{2} C S\left[A_{v}\right]+4 C S[g]+\frac{i}{4 \pi} a d A_{\mathrm{top}}\right]
\end{aligned}
$$

where the Chern-Simons terms in $a, A_{s}, A_{v}, g$ are introduced to compensate the $\eta$ invariant at the classical level (notice we have also redefined $a \rightarrow a / 2$ for later convenience), and the last term comes from the definition of

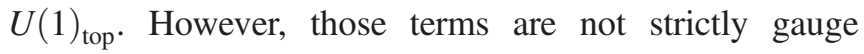
invariant in $(2+1) d$. Instead, we should consider a 4D (orientable) bulk system and view our 3D system as its boundary. The Chern-Simons terms can now be written as perfectly gauge-invariant bulk $\Theta$ terms,

$$
\begin{aligned}
S_{\text {bulk }}= & \frac{i \pi}{2}\left(p_{1}[a]+p_{1}\left[A^{s}\right]+p_{1}\left[A^{v}\right]-\sigma\left[M_{4}\right]\right. \\
& \left.+2 \int_{M_{4}} \frac{d a}{2 \pi} \wedge \frac{d A}{2 \pi}\right)
\end{aligned}
$$

where $p_{1}$ is the first Pontryagin number of the $S O(N)$ bundles [here, $a$ is viewed as an $S O(2)$ bundle],

$$
p_{1}=\frac{1}{8 \pi^{2}} \int_{M_{4}} \operatorname{tr}(F \wedge F)
$$

and $\sigma$ is the signature of the manifold, 


$$
\sigma=-\frac{1}{24 \pi^{2}} \int_{M_{4}} \operatorname{tr}(R \wedge R)
$$

where $F$ and $R$ are the gauge field strength and Riemann tensor, respectively. Both $p_{1}$ and $\sigma$ are integers. We also have the relation

$$
p_{1}=\mathcal{P}\left(w_{2}\right)+2 w_{4} \quad(\bmod 4),
$$

where $\mathcal{P}$ denotes the Pontryagin square operation, and $w_{2}$ and $w_{4}$ denote the second and fourth Stifel-Whitney classes of the $S O(N)$ bundle, respectively. Our situation is simplified by the fact that $w_{4}=0$ for $S O(2)$ and $S O(3)$ bundles. On any 2-cycle, we should have the constraint (cocycle condition)

$$
w_{2}^{a}+w_{2}^{s}+w_{2}^{v}+w_{2}^{T M}=0 \quad(\bmod 2)
$$

where $w_{2}^{a}, w_{2}^{s}, w_{2}^{v}, w_{2}^{T M}$ are the second Stifel-Whitney classes of the $a, S O(3)_{s}, S O(3)_{v}$, and tangent bundles, respectively. This condition simply comes from the fact that any field that carries half-charge under $a$ (unit charge in the notation in the main text) must also carry half-spin under $S O(3)_{s}$ and $S O(3)_{v}$, and must be a fermion. Putting the cocycle condition and Eq. (B5) into the bulk theta terms, Eq. (B2), and using the facts that (a) $\mathcal{P}[a+b]=\mathcal{P}[a]+\mathcal{P}[b]+2 a \cup b(\bmod 4)$, (b) $\mathcal{P}[a]=a \cup a(\bmod 2), \quad$ (c) $a \cup w_{2}^{T M}=a \cup a$ for $a \in H^{2}\left(M, \mathbb{Z}_{2}\right)$, (d) $\mathcal{P}\left(w_{2}^{T M}\right)=\sigma(\bmod 4)$, and (e) $w_{2}^{a}=$ $d a / 2 \pi(\bmod 2)$ for $S O(2)$ bundles, we have

$S_{\text {bulk }}=i \pi \int_{X_{4}}\left[w_{2}^{s} \cup w_{2}^{v}+\left(w_{2}^{s}+w_{2}^{v}+\frac{d A_{\text {top }}}{2 \pi}\right) \cup \frac{d A_{\text {top }}}{2 \pi}\right]$,

as promised in the main text. Notice that the bulk term only depends on the "external" gauge fields and does not depend on $a$-this is required since $a$ exists as a dynamical gauge field defined only in the $3 d$ field theory.

[1] L. Balents, Spin Liquids in Frustrated Magnets, Nature (London) 464, 199 (2010).

[2] L. Savary and L. Balents, Quantum Spin Liquids: A Review, Rep. Prog. Phys. 80, 016502 (2017).

[3] Y. Zhou, K. Kanoda, and T.-K. Ng, Quantum Spin Liquid States, Rev. Mod. Phys. 89, 025003 (2017).

[4] P. A. Lee, N. Nagaosa, and X.-G. Wen, Doping a Mott Insulator: Physics of High-Temperature Superconductivity, Rev. Mod. Phys. 78, 17 (2006).

[5] X.-G. Wen, Quantum Field Theory of Many-Body Systems: From the Origin of Sound to an Origin of Light and Electrons., Oxford Graduate Texts (Oxford University, New York, 2004).
[6] M. B. Hastings, Dirac Structure, RVB, and Goldstone Modes in the Kagomé Antiferromagnet, Phys. Rev. B 63, 014413 (2000).

[7] M. Hermele, T. Senthil, and M. P. A. Fisher, Algebraic Spin Liquid as the Mother of Many Competing Orders, Phys. Rev. B 72, 104404 (2005).

[8] Y. Ran, M. Hermele, P. A. Lee, and X.-G. Wen, ProjectedWave-Function Study of the Spin-1/2 Heisenberg Model on the Kagomé Lattice, Phys. Rev. Lett. 98, 117205 (2007).

[9] M. Hermele, Y. Ran, P. A. Lee, and X.-G. Wen, Properties of an Algebraic Spin Liquid on the Kagome Lattice, Phys. Rev. B 77, 224413 (2008).

[10] Y.-C. He, M. P. Zaletel, M. Oshikawa, and F. Pollmann, Signatures of Dirac Cones in a DMRG Study of the Kagome Heisenberg Model, Phys. Rev. X 7, 031020 (2017).

[11] Y. Iqbal, W.-J. Hu, R. Thomale, D. Poilblanc, and F. Becca, Spin Liquid Nature in the Heisenberg $J_{1}-J_{2}$ Triangular Antiferromagnet, Phys. Rev. B 93, 144411 (2016).

[12] W. Zhu, X. Chen, Y.-C. He, and W. Witczak-Krempa, Entanglement Signatures of Emergent Dirac Fermions: Kagome Spin Liquid and Quantum Criticality, Sci. Adv. 4, eaat5535 (2018).

[13] M. Hermele, T. Senthil, and M. P. A. Fisher, Algebraic Spin Liquid as the Mother of Many Competing Orders, Phys. Rev. B 72, 104404 (2005).

[14] Y. Iqbal, D. Poilblanc, and F. Becca, Spin-1/2 Heisenberg $J_{1}-J_{2}$ Antiferromagnet on the Kagome Lattice, Phys. Rev. B 91, 020402 (2015).

[15] T.-H. Han, J. S. Helton, S. Chu, D. G. Nocera, J. A. Rodriguez-Rivera, C. Broholm, and Y. S. Lee, Fractionalized Excitations in the Spin-Liquid State of a KagomeLattice Antiferromagnet, Nature (London) 492, 406 (2012).

[16] M. R. Norman, Colloquium: Herbertsmithite and the Search for the Quantum Spin Liquid, Rev. Mod. Phys. 88, 041002 (2016).

[17] Z. Zhu and S. R. White, Spin Liquid Phase of the $S=1 / 2$ $J_{1}-J_{2}$ Heisenberg Model on the Triangular Lattice, Phys. Rev. B 92, 041105(R) (2015).

[18] W.-J. Hu, S.-S. Gong, W. Zhu, and D. N. Sheng, Competing Spin-Liquid States in the Spin- $\frac{1}{2}$ Heisenberg Model on the Triangular Lattice, Phys. Rev. B 92, 140403(R) (2015).

[19] A. Wietek and A. M. Läuchli, Chiral Spin Liquid and Quantum Criticality in Extended $s=\frac{1}{2}$ Heisenberg Models on the Triangular Lattice, Phys. Rev. B 95, 035141 (2017).

[20] N. Karthik and R. Narayanan, No Evidence for Bilinear Condensate in Parity-Invariant Three-Dimensional QED with Massless Fermions, Phys. Rev. D 93, 045020 (2016).

[21] N. Karthik and R. Narayanan, Scale Invariance of ParityInvariant Three-Dimensional QED, Phys. Rev. D 94, 065026 (2016).

[22] C.-M. Jian, A. Thomson, A. Rasmussen, Z. Bi, and C. Xu, Deconfined Quantum Critical Point on the Triangular Lattice, Phys. Rev. B 97, 195115 (2018).

[23] Y. Cui, J. Dai, P. Zhou, P. S. Wang, T. R. Li, W. H. Song, J. C. Wang, L. Ma, Z. Zhang, S. Y. Li, G. M. Luke, B. Normand, T. Xiang, and W. Yu, Mermin-Wagner Physics, $(H, T)$ Phase Diagram, and Candidate Quantum SpinLiquid Phase in the Spin- $\frac{1}{2}$ Triangular-Lattice Antiferromagnet $\mathrm{Ba}_{8} \mathrm{CoNb}_{6} \mathrm{O}_{24}$, Phys. Rev. Mater. 2, 044403 (2018). 
[24] K. T. Law and P. A. Lee, 1T-TaS 2 as a Quantum Spin Liquid, Proc. Natl. Acad. Sci. U.S.A. 114, 6996 (2017).

[25] A. M. Polyakov, Quark Confinement and Topology of Gauge Theories, Nucl. Phys. B120, 429 (1977).

[26] M. Hermele, T. Senthil, M. P. A. Fisher, P. A. Lee, N. Nagaosa, and X.-G. Wen, Stability of U(1) Spin Liquids in Two Dimensions, Phys. Rev. B 70, 214437 (2004).

[27] V. Borokhov, A. Kapustin, and X. Wu, Topological Disorder Operators in Three-Dimensional Conformal Field Theory, J. High Energy Phys. 11(2002) 049.

[28] X.-Y. Song, C. Wang, A. Vishwanath, and Y.-C. He, Unifying Description of Competing Orders in Two Dimensional Quantum Magnets, Nat. Commun. 10, 4254 (2019).

[29] F. D. M. Haldane, O(3) Nonlinear $\sigma$ Model and the Topological Distinction between Integer-and Half-Integer-Spin Antiferromagnets in Two Dimensions, Phys. Rev. Lett. 61, 1029 (1988).

[30] N. Read and S. Sachdev, Spin-Peierls, Valence-Bond Solid, and Néel Ground States of Low-Dimensional Quantum Antiferromagnets, Phys. Rev. B 42, 4568 (1990).

[31] T. Senthil, A. Vishwanath, L. Balents, S. Sachdev, and M. P. A. Fisher, Deconfined Quantum Critical Points, Science 303, 1490 (2004).

[32] T. Senthil, L. Balents, S. Sachdev, A. Vishwanath, and M. P. A. Fisher, Quantum Criticality beyond the LandauGinzburg-Wilson Paradigm, Phys. Rev. B 70, 144407 (2004).

[33] J. Alicea, Monopole Quantum Numbers in the Staggered Flux Spin Liquid, Phys. Rev. B 78, 035126 (2008).

[34] J. Alicea, O. I. Motrunich, M. Hermele, and M. P. A. Fisher, Criticality in Quantum Triangular Antiferromagnets via Fermionized Vortices, Phys. Rev. B 72, 064407 (2005).

[35] Y. Ran, A. Vishwanath, and D.-H. Lee, A Direct Transition between a Neel Ordered Mott Insulator and a $d_{x^{2}-y^{2}}$ Superconductor on the Square Lattice, arXiv:0806.2321.

[36] H. C. Po, A. Vishwanath, and H. Watanabe, Complete Theory of Symmetry-Based Indicators of Band Topology, Nat. Commun. 8, 50 (2017).

[37] H. C. Po, H. Watanabe, and A. Vishwanath, Fragile Topology and Wannier Obstructions, Phys. Rev. Lett. 121, 126402 (2018).

[38] J. Cano, B. Bradlyn, Z. Wang, L. Elcoro, M. G. Vergniory, C. Felser, M. I. Aroyo, and B. A. Bernevig, Topology of Disconnected Elementary Band Representations, Phys. Rev. Lett. 120, 266401 (2018).

[39] A. Bouhon, A. M. Black-Schaffer, and R.-J. Slager, Wilson Loop Approach to Metastable Topology of Split Elementary Band Representations and Topological Crystalline Insulators with Time Reversal Symmetry, Phys. Rev. B 100, 195135 (2019).

[40] A. Thomson and S. Sachdev, Fermionic Spinon Theory of Square Lattice Spin Liquids Near the Néel State, Phys. Rev. X 8, 011012 (2018).

[41] E. Lieb, T. Schultz, and D. Mattis, Two Soluble Models of an Antiferromagnetic Chain, Ann. Phys. (N.Y.) 16, 407 (1961).

[42] M. Oshikawa, Topological Approach to Luttinger's Theorem and the Fermi Surface of a Kondo Lattice, Phys. Rev. Lett. 84, 3370 (2000).

[43] M. B. Hastings, Lieb-Schultz-Mattis in Higher Dimensions, Phys. Rev. B 69, 104431 (2004).
[44] R. Thorngren and D. V. Else, Gauging Spatial Symmetries and the Classification of Topological Crystalline Phases, Phys. Rev. X 8, 011040 (2018).

[45] M. A. Metlitski and R. Thorngren, Intrinsic and Emergent Anomalies at Deconfined Critical Points, Phys. Rev. B 98, 085140 (2018).

[46] G. Y. Cho, C.-T. Hsieh, and S. Ryu, Anomaly Manifestation of Lieb-Schultz-Mattis Theorem and Topological Phases, Phys. Rev. B 96, 195105 (2017).

[47] C.-M. Jian, Z. Bi, and C. Xu, Lieb-Schultz-Mattis Theorem and Its Generalizations from the Perspective of the Symmetry-Protected Topological Phase, Phys. Rev. B 97, 054412 (2018).

[48] V. Borokhov, A. Kapustin, and X. Wu, Topological Disorder Operators in Three-Dimensional Conformal Field Theory, J. High Energy Phys. 11 (2002) 049.

[49] T. Grover, Entanglement Monotonicity and the Stability of Gauge Theories in Three Spacetime Dimensions, Phys. Rev. Lett. 112, 151601 (2014).

[50] Y. Ran, A. Vishwanath, and D.-H. Lee, A Direct Transition between a Neel Ordered Mott Insulator and $a d_{x^{2}-y^{2}}$ Superconductor on the Square Lattice, arXiv:0806.2321.

[51] The flow to infinity is smooth with no singularity or fixed point.

[52] C. L. Kane and E. J. Mele, $\mathrm{Z}_{2}$ Topological Order and the Quantum Spin Hall Effect, Phys. Rev. Lett. 95, 146802 (2005).

[53] E. Witten, Fermion Path Integrals and Topological Phases, Rev. Mod. Phys. 88, 035001 (2016).

[54] One might wonder why our previous argument for the quantum spin Hall insulator cannot be used for the quantum valley Hall insulator-after all, the time-reversal action $\mathcal{T}_{0}$ appears to be democratic between valley and spin indices. Crucially, in our systems, the continuous valley symmetry is never exact in the UV but can only be emergent in the IR. This fact has to do with a mixed anomaly between the valley $S O(3)_{\text {valley }}$ and spin $S O(3)_{\text {spin }}$ in the continuum field theory, which is one manifestation of the parity anomaly. This means that one cannot use the previous argument here since the link between quantum spin Hall and time-reversal topological insulating behavior relies crucially on the exactness of the spin rotation symmetry-in particular, the edge-state argument assumes that there is no anomaly associated with the symmetries involved (a point dubbed "edgability" in Ref. [55]).

[55] C. Wang and T. Senthil, Boson Topological Insulators: A Window into Highly Entangled Quantum Phases, Phys. Rev. B 87, 235122 (2013).

[56] N. Karthik and R. Narayanan, Scale-Invariance and ScaleBreaking in Parity-Invariant Three-Dimensional QCD, Phys. Rev. D 97, 054510 (2018).

[57] Notice that in the $S U(4) \rightarrow S O(6)$ mapping discussed in the $\mathrm{QED}_{3}$ context, we still have a sign ambiguity due to the existence of the $S O(6)$ center $-I_{6 \times 6}$. That ambiguity is absent here since $S O(5)$ has no center.

[58] H. Song, S.-J. Huang, L. Fu, and M. Hermele, Topological Phases Protected by Point Group Symmetry, Phys. Rev. X 7, 011020 (2017).

[59] F. D. M. Haldane, Model for a Quantum Hall Effect without Landau Levels: Condensed-Matter Realization of the “Parity Anomaly”, Phys. Rev. Lett. 61, 2015 (1988). 
[60] For the edge-centered Wannier function on square and honeycomb lattices, the Wannier function is a superposition of wave functions on the endpoints of the edge.

[61] J. M. Caillol, D. Levesque, J. J. Weis, and J. P. Hansen, A Monte Carlo Study of the Classical Two-Dimensional One-Component Plasma, J. Stat. Phys. 28, 325 (1982).

[62] S. Hu, W. Zhu, S. Eggert, and Y.-C. He, Dirac Spin Liquid on the Spin-1/2 Triangular Heisenberg Antiferromagnet, Phys. Rev. Lett. 123, 207203 (2019).

[63] M. Bordelon, E. Kenney, T. Hogan, L. Posthuma, M. Kavand, Y. Lyu, M. Sherwin, C. Brown, M. J. Graf, and L. Balents, Field-Tunable Quantum Disordered Ground State in the Triangular Lattice Antiferromagnet $\mathrm{NaYbO}_{2}$, Nat. Phys. 15, 1058 (2019).

[64] Notice that this symmetry is simpler than $S O(6) \times U(1) / \mathbb{Z}_{2}$ since $S O(3) \times S O(3)$ has no center.
[65] C. Wang, A. Nahum, M. A. Metlitski, C. Xu, and T. Senthil, Deconfined Quantum Critical Points: Symmetries and Dualities, Phys. Rev. X 7, 031051 (2017).

[66] One choice of vector potential for a $2 \pi$ Dirac monopole reads $\mathbf{A}=[(1-\cos (\theta)) / 2 r \sin (\theta)] \hat{\varphi}$ in polar coordinates parametrized by $(r, \theta, \varphi)$, on the unit sphere identical to Berry connection for spin- $1 / 2$.

[67] D. V. Else, H. C. Po, and H. Watanabe, Fragile Topological Phases in Interacting Systems, Phys. Rev. B 99, 125122 (2019).

[68] S. Liu, A. Vishwanath, and E. Khalaf, Shift Insulators: Rotation-Protected Two-Dimensional Topological Crystalline Insulators, Phys. Rev. X 9, 031003 (2019).

[69] D. Gaiotto, A. Kapustin, Z. Komargodski, and N. Seiberg, Theta, Time Reversal and Temperature, J. High Energy Phys. 5 (2017) 091. 